\title{
Regularizing effects for multidimensional scalar conservation laws
}

\author{
by \\ C. CHEVERRY ${ }^{1}$ \\ CNRS UMR 6625 IRMAR, Université de Rennes I, Campus de Beaulieu, \\ 35042 Rennes Cedex, France
}

Manuscript received 8 January 1999, revised 26 June 1999

ABSTRACT. - In this article, we introduce a general method for studying the smoothing effects resulting from the non linearity in a multidimensional scalar conservation law. It turns out that the regularity of physical solutions is intimately related to a number $\Theta$ delivered after a scattering procedure. Using this approach, we recover and unify previous information while obtaining new results.

(C) 2000 L'Association Publications de l'Institut Henri Poincaré. Published by Elsevier B.V. All rights reserved

Key words: Multidimensional scalar conservation laws, Kinetic formulation of entropy solutions, Velocity averages, Scattering, Two-microlocal regularity, Radon transform

RÉSUMÉ. - Dans cet article, on s'intéresse à l'effet de lissage induit par la non linéarité dans une loi de conservation scalaire multidimensionnelle. Il se trouve que la régularité des solutions entropiques est liée à un nombre $\Theta$ obtenu à l'issue d'une procédure de scattering. Cette approche permet de retrouver, d'unifier, d'étendre et d'améliorer les résultats antérieurs. Voir Cheverry [5] pour une présentation concise en français.

(C) 2000 L'Association Publications de l'Institut Henri Poincaré. Published by Elsevier B.V. All rights reserved

\footnotetext{
${ }^{1}$ E-mail: cheverry@ maths.univ-rennes1.fr.
} 


\section{NOTATIONS}

This preliminary section is devoted to various notations that are used throughout the paper. As usual, we set:

$-a, r, s, t, \tau, \bar{\tau}, \iota$ are real numbers.

- $i, j, k, \bar{i}, \bar{j}, \bar{k}, l, m, n, N, p, q$ are natural numbers.

- $f, g, h, u, w$ are functions.

- $f \circ g$ is the non linear composition of $f$ and $g: f \circ g(x)=f(g(x))$.

$-x, y$ are points in $\mathbb{R}^{q}$ and $z, v$ are points in $\mathbb{R}$.

- $x \cdot y:=\sum_{i=1}^{q} x_{i} y_{i}$ is the inner product in $\mathbb{R}^{q}$.

- $\|x\|_{q}^{2}:=x \cdot x$ is the Euclidean norm in $\mathbb{R}^{q}$.

- $B_{q}(x, r]$ is the closed ball with center $x$ and radius $r$.

- $d m_{q}$ is the Lebesgue measure on $\mathbb{R}^{q}$.

- $|A|:=\int_{A} d m_{q}$ is the Lebesgue measure of the set $A \subset \mathbb{R}^{q}$.

- conv $A$ is the (closed) convex hull of the set $A \subset \mathbb{R}^{q}$.

- $\partial A$ is the boundary of the set $A \subset \mathbb{R}^{q}$.

- The sum $A+a$ of a set $A \subset \mathbb{R}^{q}$ and of a number $a \in \mathbb{R}^{+}$is:

$$
A+a:=\left\{x+y ; x \in A, y \in A,\|y\|_{q} \leqslant a\right\} \subset \mathbb{R}^{q} .
$$

- $\mathcal{B}(X ; Y)$ is a Banach space of functions from $X$ to $Y$.

- $\mathcal{B}(X)$ is a Banach space of functions from $X$ to $\mathbb{R}$.

- $\mathcal{B}_{c}(X ; Y)$ and $\mathcal{B}_{c}(X)$ refer to functions that have compact support.

- $\mathcal{B}_{\text {loc }}(X ; Y)$ and $\mathcal{B}_{\text {loc }}(X)$ refer to functions that are locally in $\mathcal{B}(X ; Y)$ or $\mathcal{B}(X)$.

- $\operatorname{Lip}(X ; Y)$ are the Lipschitzian functions from $X$ to $Y$.

- supp $u$ is the support of the function $u(\cdot)$.

- $C^{k}(X)$ is the space of functions in $X$ with continuous derivatives of order less than $k$.

- $\mathcal{S}\left(\mathbb{R}^{q}\right)$ is the Schwartz space of rapidly decreasing $C^{\infty}\left(\mathbb{R}^{q}\right)$ functions.

- $\mathcal{S}^{\prime}\left(\mathbb{R}^{q}\right)$ is the dual space of tempered distributions.

- $H^{\tau}\left(\mathbb{R}^{q}\right)$ is the Sobolev space of distributions with $L^{2}$ derivatives of order $\tau$. Its norm is denoted by $\|u\|_{H^{\tau}\left(\mathbb{R}^{q}\right)}$.

- $\alpha$ is a multi-index $\alpha=\left(\alpha_{1}, \ldots, \alpha_{q}\right) \in \mathbb{N}^{q}$.

$-|\alpha|$ is the length $\alpha_{1}+\cdots+\alpha_{q}$ of $\alpha$.

- $\langle\xi\rangle_{q}$ is an abbreviation for $\sqrt{1+\|\xi\|_{q}^{2}}$.

- $\partial^{\alpha}$ is the partial derivative $\partial_{1}^{\alpha_{1}} \ldots \partial_{q}^{\alpha_{q}}$ where $\partial_{j}:=\frac{\partial}{\partial y_{j}}$.

$-\nabla_{y}$ is $\left(\partial_{1}, \ldots, \partial_{q}\right)$. 
- $L^{p}\left(\mathbb{R}^{q}\right)$ is the space consisting of all measurable functions on $\mathbb{R}^{q}$ that are $p$ th-power integrable. Its norm is $\|u\|_{L^{p}\left(\mathbb{R}^{q}\right)}$.

- $W^{m, p}\left(\mathbb{R}^{q}\right)$ is the classical Sobolev space with norm $\|u\|_{W^{m, p}\left(\mathbb{R}^{q}\right)}$.

- $W^{\tau, 1}\left(\mathbb{R}^{q}\right)$ with $\left.\tau \in\right] 0,1[$ is the space of functions with norm:

$$
\|u\|_{W^{\tau, 1}\left(\mathbb{R}^{q)}\right)}:=\|u\|_{L^{1}\left(\mathbb{R}^{q}\right)}+\int_{\mathbb{R}^{q}} \int_{\mathbb{R}^{q}} \frac{|u(y)-u(\bar{y})|}{|y-\bar{y}|^{q+\tau}} d m_{q} \otimes d m_{q} .
$$

- $\mathcal{M}_{b}\left(\mathbb{R}^{q}\right)$ is the space of finite Borel measures. Its norm is:

$$
\|\mu\|_{\mathcal{M}_{b}\left(\mathbb{R}^{q)}\right)}:=\sup \left\{|\langle\mu, \varphi\rangle| ;\|\varphi\|_{C_{c}^{0}\left(\mathbb{R}^{q)}\right.} \leqslant 1\right\} .
$$

- $B V\left(\mathbb{R}^{q}\right)$ is the space of measurable functions with all distributional derivatives of order 1 that are in $\mathcal{M}_{b}\left(\mathbb{R}^{q}\right)$.

- $\operatorname{TV}_{[a, b[}(u):=\sup \left\{\sum_{j=1}^{i-1}\left|u\left(y_{j+1}\right)-u\left(y_{j}\right)\right| ; a \leqslant y_{1}<\cdots<y_{i}<b\right\}$ is the variation of $u(\cdot) \in B V([a, b[)$.

- If $u \in B V(\mathbb{R}), u(x-)$ and $u(x+)$ are the one-sided limits of $u$ at $x$.

$-u_{-} / u_{+}$designates a discontinuity separating the states $u_{-}$and $u_{+}$.

- $\mathcal{F}_{q}(u)(\xi) \equiv \hat{u}(\xi):=\int_{\mathbb{R}^{q}} \mathrm{e}^{-i y \cdot \xi} u(y) d m_{q}$ is the Fourier transform of the function $u$.

- $\left\langle D_{x}\right\rangle^{s} u(\cdot)$ is defined by $\mathcal{F}_{q}\left(\left\langle D_{x}\right\rangle^{s} u\right)(\xi)=\langle\xi\rangle_{q}^{s} \hat{u}(\xi)$.

It is now convenient to introduce some specific notations.

The symbol $C(*)$ where the star is replaced by relevant quantities stands for constants appearing in various estimates.

In what follows, $\mathbb{P}^{q}$ denotes the space of all hyperplanes in $\mathbb{R}^{q}$. Each element $\mathrm{H}_{\omega, z} \in \mathbb{P}^{q}$ can be written $\mathrm{H}_{\omega, z}:=\{y ; \omega \cdot y=z\}$ where $\omega$ is a unit vector in the sphere $\mathbb{S}^{q-1}$ and $z \in \mathbb{R}$. The $q-1$ dimensional Lebesgue measure on $\mathrm{H}_{\omega, z}$ is $d m_{\omega, z}$.

The symbol $\omega^{\perp}$ designates any vector orthogonal to $\omega$.

We fix a coordinate system:

$$
\mathbb{R}_{y}^{q} \ni y=\left(y^{\prime}, y^{\prime \prime}\right) \in \mathbb{R}_{y^{\prime}}^{r} \times \mathbb{R}_{y^{\prime \prime}}^{d}, \quad r+d=q,
$$

with dual variables:

$$
\mathbb{R}_{\xi}^{q} \ni \xi=\left(\xi^{\prime}, \xi^{\prime \prime}\right) \in \mathbb{R}_{\xi^{\prime}}^{r} \times \mathbb{R}_{\xi^{\prime \prime}}^{d} .
$$

In particular, the decomposition of $\mathbb{R}_{y}^{q}$ into a family of parallel hyperplanes with normal vector $\omega$ corresponds to the choice:

$$
\mathbb{R}_{y}^{q} \ni y=\left(y^{\prime}, y^{\prime \prime}\right)=\left(\iota \omega, \omega^{\perp}\right) \in \mathbb{R}_{y^{\prime}}^{1} \times \mathbb{R}_{y^{\prime \prime}}^{q-1}, \quad \iota \in \mathbb{R} .
$$


We will use the space:

$$
H^{\tau, \bar{\tau}}\left(\mathbb{R}_{y^{\prime}}^{r} \times \mathbb{R}_{y^{\prime \prime}}^{d}\right):=\left\{u \in \mathcal{S}^{\prime}\left(\mathbb{R}^{q}\right) ;\left\langle\xi^{\prime}\right\rangle_{r}^{\tau}\left\langle\xi^{\prime \prime}\right\rangle_{d}^{\bar{\tau}} \hat{u}(\xi) \in L^{2}\left(\mathbb{R}^{q}\right)\right\}
$$

with the Hilbert norm:

$$
\|u\|_{H^{\tau, \bar{\tau}}\left(\mathbb{R}_{y^{\prime}}^{r} \times \mathbb{R}_{y^{\prime \prime}}^{d}\right)}:=\left(\int_{\mathbb{R}^{q}}\left\langle\xi^{\prime}\right\rangle_{r}^{2 \tau}\left\langle\xi^{\prime \prime}\right\rangle_{d}^{2 \bar{\tau}}|\hat{u}(\xi)|^{2} d m_{q}\right)^{1 / 2} .
$$

We will need the following semi-norms:

$$
\begin{gathered}
|u|_{\mathcal{B}^{k}\left(\mathbb{R}^{q}\right)}:=\sum_{\left\{\gamma \in \mathbb{N}^{q} ;|\gamma|=k\right\}}\left\|\partial_{y}^{\gamma} u\right\|_{\mathcal{B}\left(\mathbb{R}^{q}\right)}, \\
|u|_{\mathcal{B}^{i, j}\left(\mathbb{R}_{y^{\prime}}^{r} \times \mathbb{R}_{y^{\prime \prime}}^{d}\right)}:=\sum_{\left\{(\alpha, \beta) \in \mathbb{N}^{r} \times \mathbb{N}^{d} ;|\alpha|=i,|\beta|=j\right\}}\left\|\partial_{y^{\prime}}^{\alpha} \partial_{y^{\prime \prime}}^{\beta} u\right\|_{\mathcal{B}\left(\mathbb{R}^{q}\right)},
\end{gathered}
$$

with the corresponding norms:

$$
\begin{gathered}
\|u\|_{\mathcal{B}^{k}\left(\mathbb{R}^{q}\right)}:=\sum_{\{\bar{k} \in \mathbb{N} ; \bar{k} \leqslant k\}}|u|_{\mathcal{B}^{\bar{k}}\left(\mathbb{R}^{q}\right)}, \\
\|u\|_{\mathcal{B}^{i, j}\left(\mathbb{R}_{y^{\prime}}^{r} \times \mathbb{R}_{y^{\prime \prime}}^{d}\right)}:=\sum_{\left\{(\bar{i}, \bar{j}) \in \mathbb{N}^{2} ; \bar{i} \leqslant i, \bar{j} \leqslant j\right\}}|u|_{\mathcal{B}^{\bar{i}, \bar{j}}\left(\mathbb{R}_{y^{\prime}}^{r} \times \mathbb{R}_{y^{\prime \prime}}^{d}\right)} .
\end{gathered}
$$

According to the preceding conventions, we have for instance the identification:

$$
\left(L^{1}\right)^{m}\left(\mathbb{R}^{q}\right) \equiv W^{m, 1}\left(\mathbb{R}^{q}\right), \quad \mathcal{M}_{b}^{1}\left(\mathbb{R}^{q}\right) \equiv B V\left(\mathbb{R}^{q}\right), \quad m \in \mathbb{N} .
$$

The Radon transform of $u$ is defined as the function $\tilde{u}$ on $\mathbb{P}^{q}$ given by the formula:

$$
\tilde{u}(\omega, z):=\int_{\mathrm{H}_{\omega, z}} u(y) d m_{\omega, z}, \quad(\omega, z) \in \mathbb{S}^{q-1} \times \mathbb{R} .
$$

The operator:

$$
\begin{aligned}
R a: L^{1}\left(\mathbb{R}^{q}\right) & \rightarrow L^{1}\left(\mathbb{P}^{q}\right) \\
u & \mapsto\left\{\begin{array}{l}
R a(u): \mathbb{P}^{q} \rightarrow \mathbb{R}, \\
(\omega, z) \mapsto \tilde{u}(\omega, z),
\end{array}\right.
\end{aligned}
$$


is a continuous linear map. An index $y$ as for:

$$
R a_{y}(u)(\omega, z, a):=\int_{\{y ; \omega \cdot y=z\}} u(y, a) d m_{\omega, z}, \quad u \in L^{\infty}\left(\mathbb{R}_{a}^{\bar{q}} ; L^{1}\left(\mathbb{R}_{y}^{q}\right)\right)
$$

indicates that the integration concerns only the $y$ variable.

Let us now state without proof (we refer for the details to the book of Helgason [13]) a few elementary properties related to the Radon transform. It is closely connected with the Fourier Transform since:

$$
\hat{u}(s \omega)=\int_{\mathbb{R}} \mathrm{e}^{-i s z} \tilde{u}(\omega, z) d z, \quad \forall(s, \omega) \in \mathbb{R} \times \mathbb{S}^{q-1}
$$

We have:

$$
R a\left(\partial_{y_{j}} u\right)(\omega, z)=\omega_{j}\left(\partial_{z} \tilde{u}\right)(\omega, z) .
$$

Moreover, the function $u$ can be recovered from its Radon transform $\tilde{u}$ by means of an explicit inversion formula (see [13, p. 72]).

It will be convenient to introduce the velocity distributions (or profiles) $\chi_{\alpha}(v)$ which are parameterized by $\alpha \in \mathbb{R}$ and that are defined by:

$$
\chi_{\alpha}(v):= \begin{cases}1 & \text { if } 0<v \leqslant \alpha \\ -1 & \text { if } \alpha \leqslant v<0 \\ 0 & \text { elsewhere }\end{cases}
$$

Finally, we introduce a family of semi-norms which are indexed by the unit vectors in the sphere $\mathbb{S}^{q-1}$ :

$$
\begin{aligned}
|u|_{\mathcal{M}_{b, \omega}^{1}(\mathbb{R})} & :=\left\|\partial_{z} \tilde{u}(\omega, \cdot)\right\|_{\mathcal{M}_{b}(\mathbb{R})}=|\tilde{u}(\omega, \cdot)|_{\mathcal{M}_{b}^{1}(\mathbb{R})} \\
& =\sup \left\{\left|\int_{\mathbb{R}^{q}} \omega \cdot \nabla_{y} u(y) \varphi(\omega \cdot y) d m_{q}\right| ;\|\varphi\|_{C_{c}^{0}(\mathbb{R})} \leqslant 1\right\} .
\end{aligned}
$$

\section{MAIN RESULTS}

We consider the initial value problem for a multidimensional scalar conservation law:

$$
\left\{\begin{array}{l}
\partial_{t} \varrho(t, x)+\sum_{i=1}^{N} \partial_{x_{i}}\left(A_{i} \circ \varrho\right)(t, x)=0, \quad(t, x) \in \mathbb{R}^{+} \times \mathbb{R}^{N}, \quad\left(\mathcal{L}_{0}^{N}\right) \\
\varrho(0, x)=\varrho_{0}(x),
\end{array}\right.
$$


where the flux $A(\cdot)$ is assumed to be sufficiently regular:

$$
A:=\left(A_{1}, \ldots, A_{N}\right) \in C^{2+n}\left(\mathbb{R} ; \mathbb{R}^{N}\right), \quad n \in \mathbb{N}_{*} .
$$

The initial data $\varrho_{0}(\cdot)$ is chosen in the space $\left(L^{\infty} \cap L^{1}\right)\left(\mathbb{R}^{N}\right)$. We set:

$$
\varrho_{0}^{\infty}:=\left\|\varrho_{0}\right\|_{L^{\infty}\left(\mathbb{R}^{N}\right)}=\sup \left\{\left|\varrho_{0}(x)\right| ; x \in \mathbb{R}^{N}\right\}<\infty .
$$

As shown by Kružkov [14], under assumptions $\left(\mathcal{H}_{1}\right)$ and $\left(\mathcal{H}_{2}\right)$, there is a unique entropy solution $\varrho(\cdot) \in C^{0}\left(\mathbb{R}_{*}^{+} ; L^{1}\left(\mathbb{R}^{N}\right)\right)$. The correspon- ding solution operator does not increase the $L^{1}$ norm. It satisfies the maximum principle. For all $t>0$ :

$$
\|\varrho(t, \cdot)\|_{L^{1}\left(\mathbb{R}^{N}\right)} \leqslant\left\|\varrho_{0}\right\|_{L^{1}\left(\mathbb{R}^{N}\right)}, \quad\|\varrho(t, \cdot)\|_{L^{\infty}\left(\mathbb{R}^{+} \times \mathbb{R}^{N}\right)} \leqslant \varrho_{0}^{\infty} .
$$

Natural questions arise concerning the smoothness of the admissible $L^{\infty}$ solutions exhibited by Kružkov [14]. Regularity is delimited on the lower end by $L^{\infty}$ and on the upper end by the inherent smoothness of general $B V$ functions, described in Vol'pert [29]. In fact, depending on the features of $A(\cdot)$, the solutions are sure to be better than $L^{\infty}\left(\mathbb{R}^{N}\right)$. They can possibly be less than $B V\left(\mathbb{R}^{N}\right)$. The task is precisely to examine the exact level of smoothness attained.

Now, without loss of generality, we can make some simplifications. Since the values $\varrho$ with $|\varrho|>\varrho_{0}^{\infty}$ are not solicited, the flux $A(\cdot)$ can be chosen to satisfy:

$$
A \in C_{c}^{2+n}\left(\mathbb{R} ; \mathbb{R}^{N}\right): \exists \eta>0 ; \operatorname{supp} A \subset B\left(0, \varrho_{0}^{\infty}+\eta\right] .
$$

Moreover the speed of propagation is finite and limited by:

$$
V:=\sup \left\{\left\|A^{\prime}(v)\right\|_{N} ;|v| \leqslant \varrho_{0}^{\infty}\right\} .
$$

Therefore, it would suffice to work locally in the space variable, with a Cauchy data that has a compact support:

$$
\varrho_{0} \in L_{c}^{\infty}\left(\mathbb{R}^{N}\right) \Rightarrow\left|\operatorname{supp} \varrho_{0}\right|<\infty .
$$

The derivative of the flux $A(\cdot)$ is the vector field denoted by:

$$
C_{c}^{1+n}\left(\mathbb{R} ; \mathbb{R}^{N}\right) \ni a(v)=\left(a_{1}(v), \ldots, a_{N}(v)\right):=A^{\prime}(v), \quad \forall v \in \mathbb{R} .
$$


We will make use of the following polarized quantities:

$$
A_{\omega}(v):=\omega \cdot A(v), \quad a_{\omega}(v):=\omega \cdot a(v), \quad \forall(v, \omega) \in \mathbb{R} \times \mathbb{S}^{N-1} .
$$

When equation $\left(\mathcal{L}_{0}^{N}\right)$ has constant coefficients:

$$
\exists a_{0} \in \mathbb{R}^{N} ; a(v)=a_{0}, \quad \forall v \in \mathbb{R},
$$

the solution $\varrho(t, x)$ is equal to $\varrho_{0}\left(x-t a_{0}\right)$. It is simply a translated function of $\varrho_{0}(\cdot)$. For all $t>0$, it is still in the space $\left(L^{\infty} \cap L^{1}\right)\left(\mathbb{R}^{N}\right)$ without any amelioration. Its regularity does not improve after resolution of $\left(\mathcal{L}_{0}^{N}\right)$.

The situation is quite different when the speed of propagation $a(\cdot)$ does depend on the state $v$ :

$$
\exists v_{0} \in \mathbb{R} ; a^{\prime}\left(v_{0}\right)=\left(A_{1}^{\prime \prime}\left(v_{0}\right), \ldots, A_{N}^{\prime \prime}\left(v_{0}\right)\right) \neq 0
$$

It is well known that the appearance of the solution $\varrho(\cdot)$ is affected by a number of dissipative mechanisms (entropy decrease, spreading of ra- refaction waves, mutual cancellation of interacting shocks with rarefaction waves ...) mostly prominent in the presence of non linearity. On account of these phenomena, the function $\varrho(\cdot)$ recovers more smoothness than the one mentioned in (1.1).

Intuitively regularizing effects are all the more marked as the acceleration $A^{\prime \prime}(\cdot)$ does not vanish a lot. In the one dimensional setting $\left(N=1, A(\cdot) \equiv A_{1}(\cdot)\right)$, it is easy to classify fluxes according to this criterion. The two (distinct) following conditions which can be imposed to a single conservation law will be of particular interest:

- The flux is strictly convex or strictly concave (the usual terminology is referred as genuine non linearity):

$$
A^{\prime \prime}(v) \neq 0, \quad \forall v \in\left[-\varrho_{0}^{\infty}, \varrho_{0}^{\infty}\right]
$$

- The flux has just one inflection point. It means that there is a unique point $i_{A}$ in $\left[-\varrho_{0}^{\infty}, \varrho_{0}^{\infty}\right]$ such as:

$$
A^{\prime \prime}(v) \neq 0, \quad \forall v \in\left[-\varrho_{0}^{\infty}, \varrho_{0}^{\infty}\right] \backslash\left\{i_{A}\right\} ; \quad A^{\prime \prime}\left(i_{A}\right)=0 .
$$

Subsequently, we will only consider non degenerate cases for which the difference

$$
\mathbb{R} \ni v \mapsto \bar{A}(v):=A\left(i_{A}+v\right)-A\left(i_{A}\right)-A^{\prime}\left(i_{A}\right), \quad v \in \mathbb{R}
$$


is subjected to (see Remarks 2.2.2 and 3.2.1):

$$
\exists v_{A}>1 ;\left|v \bar{A}^{\prime}(v)\right| \geqslant v_{A}|\bar{A}(v)|, \forall v \in\left[\varrho_{0}^{\infty}, \varrho_{0}^{\infty}\right]
$$

In the multidimensional framework, it becomes more difficult to measure how the flux $A(\cdot)$ vanishes. A natural way to proceed (in order to recover the preceding discussion) consists in projecting the function $A(\cdot)$ in each direction $\omega$. It leads to a well adapted notion:

Definition. - We define the flux $A(\cdot)$ as admissible if for all $\omega$ in $\mathbb{S}^{N-1}$ the polarized application $A_{\omega}(\cdot)$ is either subject to $(\mathcal{L D})^{1}$ or to $(\mathcal{V N} \mathcal{L})^{1}$ or to $(\mathcal{I})^{1}:=(\mathcal{I})_{1}^{1} \cup(\mathcal{I})_{2}^{1}$ (with a constant $v_{A}$ which does not depend on the directions $\omega$ in the sphere $\left.\mathbb{S}^{N-1}\right)$.

These preliminary ideas being introduced, we turn our attention to the (free) transport equation:

$$
\left\{\begin{array}{l}
\partial_{s} g(s, x, v)+a(v) \cdot \nabla_{x} g(s, x, v)=0, \quad(s, x, v) \in \mathbb{R}^{N+2}, \quad\left(\mathcal{T}_{t}^{N}\right) \\
g(t, x, v)=g_{t}(x, v)=\chi_{\varrho(t, x)}(v),
\end{array}\right.
$$

whose solution is given by the explicit formula:

$$
g(s, x, v)=g_{t}(x-(s-t) a(v), v)=\chi_{\varrho(t, x-(s-t) a(v))}(v) .
$$

Define the scattering operator:

$$
\begin{aligned}
\Xi_{t}^{N}: L^{\infty}\left(\mathbb{R}^{N}\right) & \rightarrow L^{\infty}\left(\mathbb{R}^{N+1}\right), \\
\varrho_{0} & \mapsto\left\{\begin{array}{l}
\Xi_{t}^{N}\left(\varrho_{0}\right): \mathbb{R}^{N+1} \rightarrow\{-1,0,1\}, \\
(x, v) \mapsto \chi_{\varrho(t, x+\operatorname{ta}(v))}(v) .
\end{array}\right.
\end{aligned}
$$

Its mechanism can be understood on the following diagram $(\mathcal{D})$ :

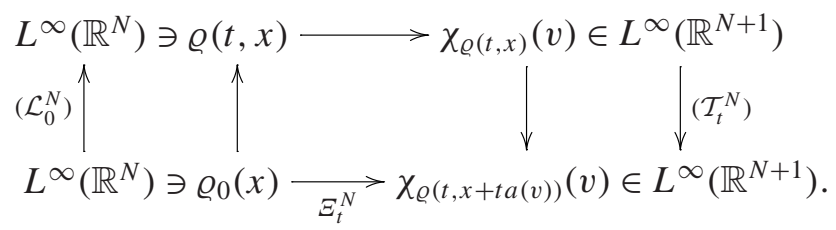

Let us introduce the number:

$$
\begin{aligned}
\Theta\left(t, \varrho_{0}\right) & :=\left|\Xi_{t}^{N}\left(\varrho_{0}\right)\right|_{\mathcal{M}_{b}^{0,1}\left(\mathbb{R}_{x}^{N} \times \mathbb{R}_{v}\right)} \\
& =\left\|\partial_{v} \Xi_{t}^{N}\left(\varrho_{0}\right)\right\|_{\mathcal{M}_{b}\left(\mathbb{R}^{N+1}\right)} \in \mathbb{R}^{+} \cup\{\infty\} .
\end{aligned}
$$


At first glance, function $\Xi_{t}^{N}\left(\varrho_{0}\right)(\cdot)$ is only bounded. Since the three top arrows of diagram $(\mathcal{D})$ preserve or diminish the $L^{1}$ norm, we easily infer:

$$
\left\|\Xi_{t}^{N}\left(\varrho_{0}\right)\right\|_{L^{1}\left(\mathbb{R}^{N+1}\right)}=\|\varrho(t, \cdot)\|_{L^{1}\left(\mathbb{R}^{N)}\right.} \leqslant\left\|\varrho_{0}\right\|_{L^{1}\left(\mathbb{R}^{N}\right)} .
$$

It turns out that the situation is even better. The construction of the operator $\Xi_{t}^{N}$ is endowed with a special compatibility property (between equations $\left(\mathcal{L}_{0}^{N}\right)$ and $\left.\left(\mathcal{T}_{t}^{N}\right)\right)$ that gives rise to a more subtle estimate. This fact is borne out by studying the case $N=1$ :

THEOREM 1.1 (Scattering in one space variable). - Assume that the flux $A(\cdot)$ is admissible. Then:

$$
\begin{array}{r}
\exists C(A) \in \mathbb{R}^{+} ; \forall \varrho_{0} \in L_{c}^{\infty}(\mathbb{R}), \forall t \in \mathbb{R}_{*}^{+}, \\
\Theta\left(t, \varrho_{0}\right) \leqslant C(A)\left|\operatorname{supp} \varrho_{0}+t\right|<\infty .
\end{array}
$$

Thanks to (1.4), it is possible to recover the previous considerations of [15-22] and to substantially improve (see Section 5.3) the results exposed in $[1-30]$.

We then present (see Section 3.3) a mild assumption $(\mathcal{H})$ (defined p. 41, it is fulfilled if for instance all the components of $A(\cdot)$ are at most quadratic) under which the majoration (1.4) extends to the general case:

THEOREM 1.2 (Multidimensional scattering). - Assume that $A(\cdot)$ is admissible and that $(\mathcal{H})$ is true. Then:

$$
\begin{gathered}
\exists C(A) \in \mathbb{R}^{+} ; \forall \varrho_{0} \in L_{c}^{\infty}\left(\mathbb{R}^{N}\right), \quad \forall t \in \mathbb{R}_{*}^{+}, \\
\Theta\left(t, \varrho_{0}\right) \leqslant C(A)\left|\operatorname{supp} \varrho_{0}+t\right|<\infty .
\end{gathered}
$$

In Section 3.3, we explain why $(\mathcal{H})$ should be systematically observed as soon as the flux $A(\cdot)$ is admissible. The demonstration of Theorem 1.2 is very significant.

Indeed, the proof shows that the bound on $\Theta\left(t, \varrho_{0}\right)$ is linked to a smoothing effect concerning the divergence of the wave speed. This aspect is established at the level of Lemma 3.3.1 and Proposition 3.3.1. In particular, under the assumptions of Theorem 1.2, we have:

$$
t\|\operatorname{div}(a \circ \varrho)(t, \cdot)\|_{\mathcal{M}_{b}\left(\mathbb{R}^{N}\right)} \leqslant C(|\operatorname{supp} \varrho(t, \cdot)|), \quad \forall t \in \mathbb{R}_{*}^{+} .
$$

The scattering process connects the non linear evolution $\left(\mathcal{L}_{0}^{N}\right)$ with the linear transport $\left(\mathcal{T}_{t}^{N}\right)$. It is worth noting that equation $\left(\mathcal{T}_{t}^{N}\right)$ is reversible. Thereby, it can also be interpreted as the Cauchy problem $\left(\mathcal{T}_{0}^{N}\right)$ whose initial data has a semi-norm $\mathcal{M}_{b}^{0,1}\left(\mathbb{R}_{x}^{N} \times \mathbb{R}_{v}\right)$ bounded by 
$\Theta\left(t, \varrho_{0}\right)$. This new point of view allows to make a synthesis of the previous lines of research relative to regularizing effects. It gives access to optimal level for the time regularity of the underlying semi-group (see Corollary 5.3.3), to Sobolev smoothness (see Corollary 5.3.4) and to twomicrolocal regularity (see below and Proposition 2.2.1).

In this summary, we point out some version of the last aspect mentioned. Let $C_{A}^{\omega}(\mathbb{R})$ be the subspace of $C^{2}(\mathbb{R})$ consisting in all functions $B(\cdot)$ that can be written in the following integral form:

$$
\exists b \in \operatorname{Lip}(\mathbb{R}) ; \quad B(\varrho)=\int_{-\infty}^{\varrho} b(v) a_{\omega}^{\prime}(v) d v, \forall \varrho \in \mathbb{R} .
$$

Now, there is a natural extension of the (classical) result due to [15-22] stating that the solution $\varrho(\cdot)$ of a convex scalar conservation law becomes instantaneously $B V(\mathbb{R})$. Indeed, in the multidimensional framework, for $t>0$, the averages with respect to some variables of well adjusted non linear expressions of the trace $\varrho(t, \cdot)$ are suitably bounded in $B V$. To be more precise:

THEOREM 1.3 (Multidimensional BV regularizing effect). - We have:

$$
\begin{aligned}
& \forall\left(A, t, \varrho_{0}, \omega, B\right) \in C^{2+n} \times \mathbb{R}_{*}^{+} \times L_{c}^{\infty} \times \mathbb{S}^{N-1} \times C_{A}^{\omega}, \exists C(A, b) \in \mathbb{R}^{+} ; \\
& |B \circ \varrho(t, \cdot)|_{\mathcal{M}_{b, \omega}^{1}(\mathbb{R})} \leqslant \frac{C(A, b)}{t}\left(\Theta\left(t, \varrho_{0}\right)+\left\|\varrho_{0}\right\|_{L^{1}\left(\mathbb{R}^{N}\right)}\right)
\end{aligned}
$$

where functions $B(\cdot)$ and $b(\cdot)$ are linked according to (1.6).

This paper is organized as follows.

Section 2 is a detailed introduction. Some complementary statements are furnished. A few historic reminders and counter-examples give a good insight into our position. Theorems 1.1 and 1.2 have to be combined. Used together, they are able to unify the former contributions of Lax [15, 16] — Dafermos [7,8] and these of Lions, Perthame and Tadmor [18].

Section 3 describes how works the operator $\Xi_{t}^{N}$. It is divided in three paragraphs. We first consider a flux which is strictly convex or strictly concave. In this particular case, the mechanism underlying diagram $(\mathcal{D})$ is simple and speaking so that Theorem 1.1 is rather easy to demonstrate. Then, we focus our attention to the other restriction on the flux (with just one non degenerate inflection point). Follows a demonstration of (1.4) which explains in concrete terms how the intricacies of the shock set are managed by the scattering process. This overcomes the main difficulty 
encountered at this stage: the possible occurrence of right contact shocks. Finally, we show (in the multidimensional setting and for admissible fluxes) that (1.5) is equivalent to some convenient bound in $\mathcal{M}_{b}\left(\mathbb{R}_{x}^{N}\right)$ for the distribution $\operatorname{div}(a \circ \varrho)(t, \cdot)$. Using the condition $(\mathcal{H})$, we can deduce (1.5). Thus, we dispose of two different (and complementary) arguments that indicate the relevance of (1.4) and (1.5).

Section 4 deals with the transport equation $\left(\mathcal{T}_{0}^{1}\right)$. We consider a special class of initial data $g_{0}(\cdot)$ that are called well prepared. The solution $g(s, \cdot)$ is integrated with respect to $v$ and we seek the regularity in the resulting variable $x \in \mathbb{R}$. This averaging technique and the restriction imposed to the Cauchy data $g_{0}(\cdot)$ allow a transfer of derivatives. It follows that the application which to $x$ associates $\int_{\mathbb{R}} g(s, x, v) d v$ has more regularity than expected.

The remainder of the article is concerned with applications. The approach of Section 4 extends to the multidimensional framework $\left(\mathcal{T}_{0}^{N}\right)$ provided that one appeals to the Radon transform. It leads to Theorem 1.3. Next, by following the method of P. Gérard [11], we deduce (see Propositions 2.2.1 and 5.2.1) a two-microlocal smoothing effect expressed in the class $H^{\tau, \bar{\tau}}$ of Hörmander. Finally, we point out other consequences. We take up the case of periodic initial data with zero mean and show sharp decay rates (Corollaries 5.3.1 and 5.3.2). In the spirit of [1] and [23], we study (see Corollary 5.3.3) the smoothness of $\varrho(\cdot)$ evaluated in the space $\operatorname{Lip}_{\text {loc }}\left(\mathbb{R}_{*}^{+} ; L^{1}\left(\mathbb{R}^{N}\right)\right)$. Following [18], we also seek the exact level of Sobolev regularity obtained for the solution.

\section{DETAILED INTRODUCTION}

\subsection{Historical reminders}

The non linearity of the flux does induce smoothing effects and decreasing large time behavior. These two aspects have been an ongoing preoccupation which dates back to Oleinik [22] and Lax [15]. Besides its intrinsic interest, this topic is connected to a fundamental issue in conservation laws such as existence theory and convergence of approximate solutions to exact solutions.

This subject has been tackled by different ways: method of characteristics $[6,7,15,17,25,30]$; compensated compactness [10,21]; semi-group point of view [1,23]; kinetic formulation and ave- raging lemma [18,28]. We will first draw a rapid picture of the matter. Then, we will attempt to unify all these approaches. 
In one space variable $\left(N=1\right.$ and $\left.A(v)=A_{1}(v)\right)$, the things are relatively well understood. We observe that the result does not share the same nature when the type of non linearity is changed.

$\mapsto(1 \sim \mathrm{i})$ The flux is strictly convex or strictly concave $-(\mathcal{V N} \mathcal{L})^{1}$ :

In such a case, solutions undergo instantaneous cancellation:

$$
\begin{gathered}
\exists C(A) \in \mathbb{R}^{+} ; \operatorname{TV}_{[x, y[}(\varrho(t, \cdot)) \leqslant C(A)(y-x) / t, \\
\forall(t, x, y) \in \mathbb{R}_{*}^{+} \times \mathbb{R}^{2}, 0<t \leqslant y-x .
\end{gathered}
$$

The bound (2.1) is mentioned for the first time in Oleinik [22]. Then, Lax $[15,16]$ considered a more specific version:

THEOREM (Lax [15, p. 23]). - Each periodic solution:

$$
L^{\infty}\left(\mathbb{R}^{+} \times \mathbb{T}\right) \ni \varrho(t, x+P)=\varrho(t, x), \quad \forall(t, x) \in \mathbb{R}^{+} \times \mathbb{R},
$$

of $\left(\mathcal{L}_{0}^{1}\right)$ satisfies sharp rates of decay in the variation of the wave speed:

$$
T V_{[x, x+P[}(a \circ \varrho(t, \cdot)) \leqslant 2 P / t, \quad \forall(t, x) \in \mathbb{R}_{*}^{+} \times \mathbb{R} .
$$

Inequality (2.1) taken with the particular choices:

$$
x=x, \quad y=x+P, \quad C(A)=2 /\left(\min _{v \in \mathbb{R}} /\left|A^{\prime \prime}(v)\right|\right)
$$

can be deduced from (2.2).

A rigorous demonstration of (2.1) in the absence of periodicity is recorded in Lax [16], Schaeffer [25] and Dafermos [7] (see also Lucier [19] who suggested another approach). Estimate (2.1) insures that the solution operator corresponding to $\left(\mathcal{L}_{0}^{1}\right)$ is compact. This particularity partly explains the interest devoted to (2.1).

$\mapsto\left(1 \sim\right.$ ii) The flux has one (non degenerate) inflection point $-(\mathcal{I})^{1}$ :

This situation was already studied in the 1980's by Benilan and Crandall [1], Dafermos [8] and Liu and Pierre [17]. These authors planed to describe the way in which the non linearity of the flux $A(\cdot)$ influences the large time behavior of solutions to $\left(\mathcal{L}_{0}^{1}\right)$. With other techniques and other purposes in mind, K. Zumbrun [30] and F. Otto [23] have recently reconsidered this question.

We refer here to the analysis of Dafermos [8] and its method of generalized characteristics that is sufficiently sharp to produce precise results. The dissipative mechanisms that affect the solution become weaker the more so as the inflection point is flatter. 
Some approximate contact discontinuity may propagate (with zero speed) in the vicinity of $\left\{(t, x) \in \mathbb{R}^{+} \times \mathbb{R} ; \varrho(t, x)=0\right\}$. The occurrence of such singularities prevents the application $\varrho(\cdot)$ from instantaneously getting a bounded variation:

Counter-example 2.1 (The single law $\left(\mathcal{L}_{0}^{1}\right)$ with $\left.A(\varrho)=\varrho^{3} / 3\right)$. - Let us consider the cubic law $\left(\mathcal{L}_{0}^{1}\right)$ associated with initial data:

$$
\varrho_{0}(x):=\left\{\begin{array}{lll}
0 & \text { if } x<0, \\
0 & \text { if } 1 /(n+1) \leqslant x<1 / n, \quad n \in 2 \mathbb{N}+1, \\
1 / n & \text { if } 1 /(n+1) \leqslant x<1 / n, \quad n \in \mathbb{N}_{*}, \\
1 & \text { if } 1 \leqslant x .
\end{array}\right.
$$

Before time $t=1 / 6$, the admissible solution $\varrho(t, \cdot)$ is composed with a sequence of shocks and rarefactions that don't touch each other:

$$
\left\{\begin{array}{lll}
0 & \text { if } x<0 \\
\sqrt{\xi} & \text { if } x=1 / n+t \xi, 0 \leqslant \xi<1 /(n-1)^{2}, & n \in 2 \mathbb{N}+1 \\
1 / n & \text { if } 1 /(n+1)+t / n^{2} \leqslant x<1 / n+t / 3 n^{2}, & n \in 2 \mathbb{N}_{*} \\
0 & \text { if } 1 / n+t / 3 n^{2} \leqslant x<1 /(n-1), & n \in 2 \mathbb{N}_{*} \\
1 & \text { if } t / 3 \leqslant x &
\end{array}\right.
$$

Due to the presence of the inflection point, the total variation of $\varrho(t, \cdot)$ remains infinite:

$$
\operatorname{TV}_{\mathbb{R}}(\varrho(t, \cdot))=1+\sum_{n=1}^{\infty} \frac{1}{n}=+\infty, \quad \forall t \in[0,1 / 6[.
$$

The total variation of higher moments that is bounded at $t=0$ is not changed. For all $k \geqslant 2$, one has:

$$
\operatorname{TV}_{\mathbb{R}}\left(\varrho(t, \cdot)^{k}\right)=1+\frac{1}{2^{k-1}} \sum_{n=1}^{\infty} \frac{1}{n^{k}}<+\infty, \quad \forall t \in[0,1 / 6[
$$

Condition $(\mathcal{I}){ }_{1}^{1}$ isolates the point $i_{A}$ in the state space. Elsewhere, we find again the genuine non linearity restriction $(\mathcal{V N} \mathcal{L})^{1}$ and the corresponding smoothing effect. Thereby, it is logical to recover the analogue of (2.1) on condition that one erases what occurs in a neighborhood of the set $\left\{(t, x) \in \mathbb{R}^{+} \times \mathbb{R} ; \varrho(t, x)=0\right\}$. This point of view is implicitly exploited in a statement of Dafermos [8]. For the sake of completeness, we record it: 
TheOrem (Dafermos $[8$, p. 232]). - The expression $D \circ \varrho(\cdot)$ where $D(\cdot)$ is the conjugate function of $A(\cdot)$ that is:

$$
D(\varrho):=\varrho A^{\prime}(\varrho)-A(\varrho)+A(0)=\int_{0}^{\varrho} v a^{\prime}(v) d v, \quad \forall \varrho \in \mathbb{R},
$$

gains the $B V(\mathbb{R})$ regularity. More precisely:

$$
\operatorname{TV}_{\mathbb{R}}(D \circ \varrho(t, \cdot)) \leqslant 2\left\|\varrho_{0}\right\|_{L^{1}(\mathbb{R})} / t, \quad \forall t \in \mathbb{R}_{*}^{+} .
$$

Remark 2.1. - For the cubic law, we get: $D(\varrho)=2 \varrho^{3} / 3$. In general, on combining definition $(2.5)$ and property $(\mathcal{I})^{1}$, we deduce:

$$
\limsup _{0 \neq \varrho \rightarrow 0}\left|D(\varrho) / \varrho^{3}\right|<\infty .
$$

We observe that a behavior similar to (2.7) does not take into account what happens in the state space near the origin. Let $\varrho_{-}$and $\varrho_{+}$be two states (whose values approach zero) connected by a shock. According to (2.7), the jump $\varrho_{-} / \varrho_{+}$contributes to (2.6) in accordance with an amount that is equivalent to:

$$
\sim \max \left(\left|\varrho_{-}\right| ;\left|\varrho_{+}\right|\right)^{2}\left|\varrho_{+}-\varrho_{-}\right| \ll\left|\varrho_{+}-\varrho_{-}\right| .
$$

This comparison implies:

$$
\nexists C \in \mathbb{R}_{*}^{+} ; \mathrm{TV}_{\mathbb{R}}(D \circ \varrho) \geqslant C \mathrm{TV}_{\mathbb{R}}(\varrho), \forall \varrho \in B V(\mathbb{R}) .
$$

It is in agreement with Counter-example 2.1. It shows that estimate (2.1) is inaccessible under the single knowledge of (2.6).

$\mapsto\left(1 \sim\right.$ iii) Any non linear flux $(\mathcal{N} \mathcal{L})^{1}$ : This situation is (for $\left.N=1\right)$ the most general. For example, it includes the case of a flux function $A(\cdot)$ whose second derivative is identically equal to zero on an open interval of $\mathbb{R}$ or the case of an application $A(\cdot)$ that has many inflection points. It combines some aspects that are regularizing and others that are not. The compensated compactness (developed by Tartar [27] and Murat [21]) brings here qualitative indications. The Young measure $v_{t, x}(\lambda)$ associated to some extracted sequence $\left(\varrho_{j}(t, x)\right)_{j \in \mathbb{N}}$ of uniformly bounded solutions of $\left(\mathcal{L}_{0}^{1}\right)$ reduces to a Dirac mass at each point where the flux $A(\cdot)$ is genuinely non linear. It implies that the sequence $\left(a \circ \varrho_{j}(t, x)\right)_{j \in \mathbb{N}}$ 
converges strongly in $L^{1}\left(\mathbb{R}^{N}\right)$. Beyond that, it does not produce any quantitative information. Some estimates generalizing (2.6) are needed here. The purpose of Theorem 1.3 is precisely to remedy to this gap.

In the multidimensional setting $(N \geqslant 2)$, the information on regularity recorded in the literature are far from definitive. Let us refer to progresses in this field.

$\mapsto(2 \sim$ i) $A$ rather negative sign: An easy computation due to Conway [6] indicates that the solution $\varrho(t, \cdot)$ can not become $B V\left(\mathbb{R}^{N}\right)$ for an acceleration vector even if it is submitted to:

$$
a^{\prime}(v) \neq 0, \quad \forall v \in \mathbb{R} .
$$

More exactly, in the context faced by Conway [6], there is one direction $\omega_{0}$ in $\mathbb{S}^{N-1}$ adjusted in such a way that (the flux is called linearly degenerate in the direction $\omega_{0}$ ):

$$
a_{\omega_{0}}^{\prime}(v)=\omega_{0} \cdot a^{\prime}(v)=0, \quad \forall v \in \mathbb{R} .
$$

It follows that for all $t>0$, the application

$$
\mathbb{R} \ni s \mapsto \varrho\left(t, x+s \omega_{0}\right) \in \mathbb{R}
$$

is for almost every $x$ in $\mathbb{R}^{N}$ in $L^{\infty}(\mathbb{R})$ and not better. It means that both the loss of convexity and the addition of space variables (these two occurrences being linked together) reduce the regularizing effects.

$\mapsto(2 \sim$ i) A rather positive sign: Let us now refer to:

Theorem (Lions, Perthame and Tadmor [18, p. 179]). - Under the non stationary constraint

$$
\begin{gathered}
\left.\left.\left.\left.\exists C \in \mathbb{R}^{+}, \exists \alpha \in\right] 0,1\right] ; \forall \delta \in\right] 0,1\right], \forall(\tau, \omega) \in \mathbb{S}^{N}, \\
m_{1}\left(\left\{v ;|v| \leqslant \varrho_{0}^{\infty},\left|\tau+a_{\omega}(v)\right| \leqslant \delta\right\}\right) \leqslant C \delta^{\alpha},
\end{gathered}
$$

the solution $\varrho(t, \cdot)$ has the Sobolev regularity:

$$
\varrho(t, \cdot) \in W^{\tau, 1}\left(\mathbb{R}^{N}\right), \quad \forall \tau \in\left[0, \alpha /(\alpha+2)\left[, \quad \forall t \in \mathbb{R}_{*}^{+} .\right.\right.
$$

We present below an outline of the analysis of Lions, Perthame and Tadmor [18]. Their method relies essentially on two notions:

(1): The kinetic formulation of equation $\left(\mathcal{L}_{0}^{N}\right)$ introduced by Perthame and Tadmor [24]. It substitutes for $\left(\mathcal{L}_{0}^{N}\right)$ a transport equation with a 
source term

$$
\left\{\begin{array}{l}
\partial_{t} f+a(v) \cdot \nabla_{x} f=\partial_{v} m \quad \text { in } \mathcal{S}^{\prime}\left(\mathbb{R}_{*}^{+} \times \mathbb{R}^{N+1}\right), \\
f(0, x, v)=\chi_{\varrho_{0}(x)}(v),
\end{array}\right.
$$

that must be completed by the two following constraints:

$$
\begin{aligned}
& f(t, x, v)=\chi_{\varrho(t, x)}(v) \quad \text { for some } \varrho \in L^{\infty}\left(\mathbb{R}_{*}^{+} \times \mathbb{R}^{N}\right) . \\
& 0 \leqslant m(t, x, v) \in \mathcal{M}_{b}\left(\mathbb{R}_{*}^{+} \times \mathbb{R}^{N+1}\right) \text { has compact support. }
\end{aligned}
$$

(2): The velocity averaging principle in the version presented by DiPerna, Lions and Meyer [9].

Please note that the method following the steps (1) and (2) leads to a regularity level $\alpha /(\alpha+2)$ which is definitely not optimal with respect to the order of Besov space:

Example 2.1. - For $N=1$, the condition $(\mathcal{N S})^{1}$ taken with $\alpha=1$ is equivalent to $(\mathcal{V} \mathcal{N} \mathcal{L})^{1}$. The number $\tau$ given by $(2.9)$ is less than $1 / 3$ (i.e., far from the foreseen value 1 obtained in (2.1)).

The step (2.9) is important since it is sufficient to guarantee the compacity in $L^{1}\left(\mathbb{R}^{N}\right)$ of the operator solution and thereby it allows to pass to the limit in non linear terms. According to this standpoint, it is decisive. However there are still things lacking. First, the condition $(\mathcal{N S})^{N}$ is rather restrictive. Anyway, it is not sufficiently precise to take separately into account what occurs in each direction $\omega$ of $\mathbb{R}_{x}^{N}$. Moreover, according to Example 2.1, the conclusion (2.9) applied with $N=1$ and $(\mathcal{V} \mathcal{N} \mathcal{L})^{1}$ does not recover fully the classical result of Oleinik [22].

The solution $f(\cdot)$ of $\left(\mathcal{C}_{0}^{N}\right)$ can be decomposed into $f_{C}(\cdot)$ plus $f_{S}(\cdot)$. Here, $f_{C}(\cdot)$ is the solution of $\left(\mathcal{C}_{0}^{N}\right)$ without the source term $\partial_{v} m(\cdot)$ but with the initial condition $\chi_{\varrho_{0}(\cdot)}(\cdot)$ whereas $f_{S}(\cdot)$ is the solution of $\left(\mathcal{C}_{0}^{N}\right)$ solved globally in space-time with the second member $\partial_{v} m(\cdot)$ but without the Cauchy data $\chi_{\varrho_{0}(\cdot)}(\cdot)$. The function $f(\cdot)$ owns at least the minimal regularity of $f_{C}(\cdot)$ and $f_{S}(\cdot)$. The term $\partial_{v} m(\cdot)$ is apparently the worst. For this reason, the attention in [18] is turned towards $f_{S}(\cdot)$. Now, the source term $\partial_{v} m(\cdot)$ is removed by applying the scattering operator. Therefore, the component $f_{C}(\cdot)$ is the one that becomes determinant regarding the regularity. In fact, the problem is pushed elsewhere. The question is now to identify constraints on $g_{0}(\cdot)$ in order to recover at the time $t$ quantitative informations on $\varrho(t, \cdot)$. Such a program is described in the next paragraph. In the frame of mind of [18], it gives access to a better level of Sobolev regularity (see Corollary 5.3.4). This leads also to a new 
interpretation (that is quite sharp) of questions relative to regularity in hyperbolic conservation laws.

\subsection{An interesting case}

We observe that the conditions previously imposed on the flux allow the scalar product $a_{\omega}^{\prime}(v)$ to vanish. For instance, it is systematic under $(\mathcal{L D})_{\omega}^{N}$ and it is taken into account by $(\mathcal{N S})^{N}$. In fact, when $N \geqslant 2$, it is guaranteed to happen:

$$
\forall v \in \mathbb{R}, \exists \omega \in \mathbb{S}^{N-1} ; a_{\omega}^{\prime}(v)=0 \quad\left(\omega \in a^{\prime}(v)^{\perp}\right) .
$$

Hence, for $N \geqslant 2$, parameters $\alpha$ and $\tau$ involved in $(\mathcal{N S})^{N}$ and (2.9) are necessarily limited by:

$$
\alpha \leqslant 1 / 2, \quad \tau<1 / 5 .
$$

We pick $\bar{\omega}$ in such a way that: $\exists v \in \mathbb{R} ; \bar{\omega} \cdot a^{\prime}(v)=0$. The search for solutions of the form $\varrho(t, x)=\rho(t, \bar{\omega} \cdot x)$ where $\rho \in L^{\infty}\left(\mathbb{R}^{+} \times\right.$ $\mathbb{R})$ gives rise to the non linear evolution problem $\left(\mathcal{L}_{0}^{1}\right)$ built with the polarized application $A_{\bar{\omega}}(\cdot)$. Therefore, we necessarily have to deal with the situation $(1 \sim$ ii) or $(1 \sim$ iii $)$. On account of Counter-example 2.1, this remark means that, under hypothesis $\left(\mathcal{H}_{2}\right)$ and for any flux $A(\cdot)$, the space $B V\left(\mathbb{R}^{N}\right)$ is certainly not available for the trace $\varrho(t, \cdot)$.

The picture is even more disadvantageous since it cannot be directly restored by non linear composition:

Counter-example 2.2.-

LEMMA 2.2.1. - Let $B(\cdot)$ be some non vanishing function in $C^{1}(\mathbb{R})$. Then:

$$
\forall t \in \mathbb{R}_{*}^{+}, \exists \varrho_{0} \in L_{c}^{\infty}\left(\mathbb{R}^{N}\right) ;\|B \circ \varrho(t, \cdot)\|_{B V\left(\mathbb{R}^{N}\right)}=+\infty
$$

Proof of Lemma 2.2.1. - Define:

$$
\diamond:=\left\{v ; B^{\prime}(v) \neq 0\right\} \cap\left\{v ; A^{\prime \prime}(v) \neq 0\right\} .
$$

We distinguish two cases:

- (1) $\diamond=\emptyset$ : Let $[c, d]$ with $c<d$ some closed interval contained in the interior of the support of $B^{\prime}(\cdot)$. There is always a function $\varrho_{0}(\cdot)$ with:

$$
\varrho_{0}(x) \in[c, d], \quad \forall x \in \mathbb{R}^{N}, \quad\left\|B \circ \varrho_{0}\right\|_{B V\left(\mathbb{R}^{N}\right)}=+\infty .
$$


As the function $a(\cdot)$ is constant on $[c, d]$, the transport is linear

$$
B \circ \varrho(t, x)=B \circ \varrho_{0}(x-\operatorname{ta}(c)), \quad \forall x \in \mathbb{R}^{N} .
$$

Hence we easily get (2.14).

$-(2) \diamond \neq \emptyset$ : Let us fix $t$ in $\mathbb{R}_{*}^{+}$. Let $[c, d]$ with $c<d$ be some closed interval included in the open set $\diamond$. We choose a point $\bar{v}$ in $] c, d$ [ and a direction $\bar{\omega}$ orthogonal to the vector $A^{\prime \prime}(\bar{v})$. We can now sketch the proof. By reproducing for the flux $\bar{\omega} \cdot A(\cdot)$ in the neighborhood of $\bar{v}$ in the state space the construction made at the level of Counter-example 2.1, we produce some (one dimensional) solution $\varrho(\cdot)$ such that:

$$
\varrho(t, x)=\rho(t, \omega \cdot x) \in[c, d], \quad \forall x \in \mathbb{R}^{N}, \quad\|\rho(t, \cdot)\|_{B V(\mathbb{R})}=+\infty .
$$

It implies (since the derivative $B^{\prime}(\cdot)$ does not vanish on $[c, d]$ ):

$$
\|B \circ \varrho(t, \cdot)\|_{B V\left(\mathbb{R}^{N}\right)} \sim\|\rho(t, \cdot)\|_{B V(\mathbb{R})}=+\infty .
$$

In view of (2.14), an inequality analogous to this of Dafermos (with $\mathbb{R}$ replaced by $\mathbb{R}^{N}$ ) is certainly not accessible. Since, in another connection, Lions, Perthame and Tadmor [18] observe a little smoothing effect, the problem is to know what is the regularity exactly reached and how it expresses itself. We will bring a few details relative to these two questions.

The notion of scattering is essential in various contexts (non linear wave equation, Schrödinger's equation, diffusion ...). At first sight, its relevance to our theme can be surprising.

Now, it was already present at the beginning. In his pioneering work, in order to get (2.2), Lax [15] returned to time $t=0$ by following backward characteristics. Modern regularizing theory has partially left behind this old method using characteristics. However, the short advance of Lax [15] admits a more abstract formulation intending to absorb the most general situations. It consists in passing through the diagram $(\mathcal{D})$. This transfers the problem towards the search of the uniform bound (1.5). We will establish (1.5) for a large class of fluxes (Theorems 1.1 and 1.2). One is tempted to conjecture that (1.5) is true without any assumption on $A(\cdot)$.

When equation $\left(\mathcal{L}_{0}^{N}\right)$ has constant coefficients or when the solution of $\left(\mathcal{L}_{0}^{N}\right)$ is smooth up to time $t$, we find that $\Xi_{t}^{N}\left(\varrho_{0}\right)(\cdot)$ coincides with $\chi_{\varrho_{0}(\cdot)}(\cdot)$. As a result:

$$
\Theta\left(t, \varrho_{0}\right)=\left\|\delta_{\varrho_{0}}-\delta_{0}\right\|_{\mathcal{M}_{b}\left(\mathbb{R}_{x}^{N} \times \mathbb{R}_{v}\right)}=2\left|\operatorname{supp} \varrho_{0}\right|
$$


Thus, we observe that, under the condition $(\mathcal{L D})^{N}$ or for regular solutions, inequality (1.5) is satisfied with $C(A)=2$. The bound (1.5) expresses a compatibility property between $\left(\mathcal{L}_{0}^{N}\right)$ and $\left(\mathcal{T}_{t}^{N}\right)$. The equation $\left(\mathcal{L}_{0}^{N}\right)$ can be interpreted in view of $\left(\mathcal{C}_{0}^{N}\right)$. The constraint $(2.10)$ comes from the so-called "transport-collapse operator" introduced by Brenier [4]. It prevents the solution $f(\cdot)$ of the transport equation $\left(\mathcal{C}_{0}^{N}\right)$ from becoming multivalued. In particular, it implies:

$$
|f(t, \cdot)|_{\mathcal{M}_{b}^{0,1}\left(\mathbb{R}_{x}^{N} \times \mathbb{R}_{v}\right)}=2|\operatorname{supp} \varrho(t, \cdot)|<\infty .
$$

Theorems 1.1 and 1.2 state that the return to the initial time thanks to $\left(\mathcal{T}_{t}^{N}\right)$ does not change the boundedness noted line (2.16) precisely because the trace $f(t, \cdot)$ has conveniently been prepared by the non linear evolution $\left(\mathcal{L}_{0}^{N}\right)$. This last hypothesis is very important. $\operatorname{Under}(\mathcal{N} \mathcal{L})^{N}$, the bound (1.5) is unstable. When the trace $\varrho(t, \cdot)$ is replaced by any bounded function which does not descend from the resolution of $\left(\mathcal{L}_{0}^{N}\right)$, the bound (1.5) is violated:

$$
\begin{gathered}
\forall C \in \mathbb{R}_{*}^{+}, \forall s \in \mathbb{R} \backslash\{t\}, \exists \varrho \in L_{c}^{\infty}\left(\mathbb{R}^{N}\right) ; \\
\left|\chi_{\varrho(x-(s-t) a(v))}(v)\right|_{\mathcal{M}_{b}^{0,1}\left(\mathbb{R}_{x}^{N} \times \mathbb{R}_{v}\right)} \geqslant C .
\end{gathered}
$$

Inequality $(1.4)$ is true under $(\mathcal{V N} \mathcal{L})^{1}$. In this particular case, this result is a trivial consequence of a property given at Proposition 3.1.1. It is satisfied under $(\mathcal{I})^{1}$. Under $(\mathcal{H})$, this is also guaranteed for a large class of multidimensional fluxes (see Remark 3.2.2). Thus, it is now clear that the operator $\Xi_{t}^{N}$ is a key to the understanding of the smoothing effects induced by non linearity. Using this operator allows to transcribe all the non linear informations $\left(\mathcal{L}_{0}^{N}\right)$ towards the linear model $\left(\mathcal{T}_{t}^{N}\right)$ and thereby to simplify notably the analysis.

The point is now to understand at the right of diagram $(\mathcal{D})$ how some control on $\Theta\left(t, \varrho_{0}\right)$ leads to a gain of regularity after averaging in $v$. With respect to the two-microlocal point of view, such a progress is not systematic. It only appears when the initial condition $g_{0}(\cdot)$ is well adjusted. The manipulation preparing the Cauchy data $g_{0}(\cdot)$ gives rise, after integration with respect to $v$, to the advent of the non linear expressions $B \circ \varrho(\cdot)$ with $B(\cdot)$ chosen in the space $C_{A}^{\omega}(\mathbb{R})$. These considerations underline the importance of two complementary ingredients which are interdependent in view of Counter-example 2.2, the averaging procedure and the non linear composition. In Theorem 1.3 , the geometry is quite rigid in so far as the averages are taken along 
a fixed family of hyperplanes (the same result can be expressed more intrinsically by integrating along the leaves of a regular foliation). Once such decomposition of the space variable $\mathbb{R}_{x}^{N}$ is fixed, we can adapt to it the non linear compositions by choosing appropriately the diverse functions $B(\cdot)$. We can clarify the link between notions of averaging and composition. The relevant applications $B(\cdot)$ are collected in the set $C_{A}^{\omega}(\mathbb{R})$. What is important here is the flatness of $B(\cdot)$ in the vicinity of zero. Following this idea, the equivalent definition:

$$
C_{A}^{\omega}(\mathbb{R}) \equiv C_{A_{\omega}}^{1}(\mathbb{R})=\left\{B ; B^{\prime} \in a_{\omega}^{\prime} \times \operatorname{Lip}(\mathbb{R})\right\} \subset C^{2}(\mathbb{R})
$$

is perhaps more suggestive since it brings out the singularity of $A_{\omega}(\cdot)$. Let us now describe more carefully the elements $B(\cdot)$ allowed when different behaviors of the flux $v \mapsto A(v)$ are taken into account.

$-C_{A}^{\omega}(\mathbb{R})$ reduces to $\{0\}$ in case of $(\mathcal{L D})_{\omega}^{N}$. Thus, under $(\mathcal{L D})^{N}$, one has $C_{A}^{\omega}(\mathbb{R})$ equal to $\{0\}$ for all angles $\omega$ in the sphere $\mathbb{S}^{N-1}$.

- It is always a non trivial subspace $\left(C_{A}^{\omega}(\mathbb{R}) \neq\{0\}\right)$ when:

$$
\exists v \in \mathbb{R} ; a_{\omega}^{\prime}(v) \neq 0 .
$$

$(\mathcal{N} \mathcal{L})_{\omega}^{N}$

- When the polarized flux $A_{\omega}(\cdot)$ is subject to $(\mathcal{I})^{1}$, the set $C_{A}^{\omega}(\mathbb{R})$ contains the conjugate function of Dafermos [8] (choose $b(\cdot)$ defined by $b(v)=v$ if $v>0$ and $b(v)=0$ if $v \leqslant 0$; plug this $b(\cdot)$ into (1.6) and then compare with (2.5)) but also a number of other expressions. Some of them inherit a behavior at the origin less flat than the one observed with $D(\cdot)$. For example, the polarized speed $a_{\omega}(\cdot)$ corresponding to the choice $b(\cdot) \equiv 1$ does satisfy a better estimation than (2.7) since:

$$
\limsup _{\varrho \rightarrow 0}\left|a_{\omega}(\varrho)\right| / \varrho^{2}<\infty .
$$

- Let us assume that:

$$
a_{\omega}^{\prime}(v) \neq 0, \quad \forall v \in \mathbb{R} . \quad(\mathcal{V N} \mathcal{L})_{\omega}^{N}
$$

Then, the restriction $C_{A}^{\omega}(\mathbb{R}) \rightarrow W^{2, \infty}\left(\left[-\varrho_{0}^{\infty} ; \varrho_{0}^{\infty}\right]\right)$ is surjective. It means that all non linear compositions (and in particular the linear one $v \mapsto v)$ are allowed. However, note that condition $(\mathcal{V N} \mathcal{L})_{\omega}^{N}$ never happens for all the directions in the sphere $\mathbb{S}^{N-1}$ (see (2.12)). The lack of $B V$ regularity occurs at this particular level.

The control on $\Theta\left(t, \varrho_{0}\right)$ does depend on the measure of the support of $\varrho_{0}(\cdot)$ but not on the regularity of $\varrho_{0}(\cdot)$ (beyond $L^{1}\left(\mathbb{R}^{N}\right)$ ). In order 
to establish (1.7), it is sufficient to get a bound that is uniformly valid for a dense subset (for instance $\left.B V\left(\mathbb{R}^{N}\right)\right)$ of $\left(L^{1} \cap L^{\infty}\right)\left(\mathbb{R}^{N}\right)$. This way, the regularizing effects are evaluated at the level of "smooth" functions yet. It is much more practical and more precise to work with $B V\left(\mathbb{R}^{N}\right)$ solutions than directly with $L^{\infty}\left(\mathbb{R}^{N}\right)$ solutions. This explains partly why the method is powerful and leads to the quantitative estimates (1.7). These last ones are sharper than the informations obtained by compensated compactness.

The reader should also note that function $g_{0}(\cdot)$ (in case of weak solutions) is completely different from $\chi_{\varrho_{0}}(\cdot)$. This change of initial data can be surprising. When you come to think of it, it is coherent with our purpose (and with the observation (2.1) of Lax [15]) since the controls (1.4), (1.5) and therefore (1.7) do not depend on the features of $\varrho_{0}(\cdot)$ (besides its $L^{1}$-norm that after all is preserved by the scattering procedure: see (1.3)). Thus, it is justified to concentrate on the right of diagram $(\mathcal{D})$. Theorem 1.3 is established under this perspective and captures the essential features of the regularity of solutions. Its strength is illustrated below by a succession of remarks.

Remark 2.2.1. - Let us interpret (1.7) when $N=1$. We have:

$$
\mathbb{S}^{0}=\{-1,1\}, \quad \tilde{\varrho}(-1, z)=\varrho(-z), \quad \tilde{\varrho}(1, z)=\varrho(z) .
$$

Hence, Statement 1.3 says that:

$$
\begin{aligned}
\operatorname{TV}_{\mathbb{R}}\left(R a_{x}(B \circ \varrho)(t, 1, \cdot)\right) & =\operatorname{TV}_{\mathbb{R}}\left(R a_{x}(B \circ \varrho)(t,-1, \cdot)\right) \\
& =\operatorname{TV}_{\mathbb{R}}(B \circ \varrho(t, \cdot)) \\
& \leqslant C(B)\left(\Theta\left(t, \varrho_{0}\right)+\left\|\varrho_{0}\right\|_{L^{1}(\mathbb{R})}\right) / t
\end{aligned}
$$

for all functions $B(\cdot)$ satisfying:

$$
\exists b \in \operatorname{Lip}(\mathbb{R}), \quad B(\varrho)=\int_{-\infty}^{\varrho} b(v) a^{\prime}(v) d v, \forall \varrho \in \mathbb{R} .
$$

Under $(\mathcal{V N} \mathcal{L})^{1}$, according to (1.4), bound (2.18) is the same as (2.1). In this very particular case, our method rediscovers in an elegant way the results of Oleinik [22] and Lax [15,16].

Under $(\mathcal{I})^{1}$, the control (2.18) extends (2.6) and gives access to sharper informations. To be convinced of this fact, just compare the limits (2.7) and (2.17). The progress can be illustrated easily in view of Counterexample 2.1. Dafermos [8] allows the powers $k \geqslant 3$ whereas Theorem 1.1 
combined with (2.18) gives $k \geqslant 2$. For further results in this direction, see the Corollaries 5.3.2 and 5.3.3.

Under the general hypothesis $(\mathcal{N} \mathcal{L})^{1}$, the bound $(2.18)$ puts in concrete form the program drawn in $(1 \sim$ iii $)$.

The contents of Theorems 1.1, 1.2 and 1.3 in the multidimensional framework $(N \geqslant 2)$ is more difficult to understand. Their novelty and their interest can be founded by the following considerations:

Remark 2.2.2. - For homogeneous fluxes $A(v)=c v^{m+1}$ with $m \geqslant 2$, we find:

$$
v A^{\prime}(v) / A(v)=m+1=v_{A} \geqslant 3, \quad \forall v \in\left[-\varrho_{0}^{\infty}, \varrho_{0}^{\infty}\right] .
$$

Now, an inflection point is generically not flat. In general, it is adjusted so that:

$$
\exists m_{\omega} \in[2,1+n] ; \quad A_{\omega}^{\left(m_{\omega}+1\right)}\left(i_{A_{\omega}}\right) \neq 0,
$$

where $m_{\omega}$ is related to the order of annulation of $A_{\omega}(\cdot)$ at position $i_{A_{\omega}}$ :

$$
A_{\omega}^{(j)}\left(i_{A_{\omega}}\right)=0, \quad \forall j \in\left\{0, \ldots, m_{\omega}\right\} .
$$

Condition (2.19) implies $(\mathcal{I})^{1}$ (see the Remark 3.2.1). Thus, the constraint imposed on the flux in Theorems 1.1 and 1.2 is not at all restrictive. It is globally verified for all polynomial (vector valued) fluxes $A(\cdot)$ of degree less than three. For $m=0$, we have only to consider $(\mathcal{L D})^{1}$. For $m=1$, we have to deal both with $(\mathcal{L D})^{1}$ and with $(\mathcal{V N} \mathcal{L})^{1}$. For $m=2$, the three possibilities $(\mathcal{L D})^{1},(\mathcal{I})^{1}$ and $(\mathcal{V N} \mathcal{L})^{1}$ can occur.

The admissibility criterion is locally (near each fixed $\varrho$ in the state space) satisfied by the flux $A(\cdot)$ as long as:

- there is no accumulation of inflection points: $\exists \varepsilon \in \mathbb{R}_{*}^{+}$such that $\forall \omega \in$ $\mathbb{S}^{N-1}$, either we have $(\mathcal{L D})^{1}$ either there exists at most one inflection point $i_{A_{\omega}} \in[\bar{\varrho}-\varepsilon, \bar{\varrho}+\varepsilon]$;

- there is no $C^{\infty}$ flatness: $\forall \omega \in \mathbb{S}^{N-1}$, if $(\mathcal{I})^{1}$ is true on the interval $[\bar{\varrho}-\varepsilon, \bar{\varrho}+\varepsilon]$ then $\exists m_{\omega} \in \mathbb{N}$ such that $A_{\omega}^{\left(m_{\omega}+1\right)}\left(i_{A_{\omega}}\right) \neq 0$.

Please note also that if (2.19) is correct for all directions $\omega$ in $\mathbb{S}^{N-1}$, the indice $\alpha$ involved by $(\mathcal{N S})^{N}$ is exactly $\left(\inf _{\omega \in \mathbb{S}^{N-1}} 1 / m_{\omega}\right)$.

Remark 2.2.3. - The estimate (1.7) is optimal in its formulation. It means that the regularity in $z$ of the application $R a_{x}(B \circ \varrho)(t, \omega, z)$ cannot go beyond what is announced in (1.7). It is clear when compared with the one dimensional solutions $\rho(t, \omega \cdot x)$. Indeed, we find again the 
case $\left(\mathcal{L}_{0}^{1}\right)$. We then know that the $B V(\mathbb{R})$ regularity cannot be improved (because of the formation of shocks).

Remark 2.2.4. - Let us suppose to simplify the discussion that the first component $A_{1}(\cdot)$ is strictly convex (or strictly concave) and let us fix the direction $\omega_{1}:=(1,0, \ldots, 0)$. The polarized flux $A_{\omega_{1}}(\cdot)$ then coincides with $A_{1}(\cdot)$ and the space $C_{A}^{\omega_{1}}(\mathbb{R})$ can (locally) be identified with $W^{2, \infty}(\mathbb{R})$. The Radon transform evaluated for the angle $\omega_{1}$ is simply the integration with respect to the $N-1$ last variables

$$
x^{\prime \prime}=\left(x_{2}, \ldots, x_{N}\right), \quad d x^{\prime \prime}=d x_{2} \ldots d x_{N} .
$$

It gives rise to the action:

$$
\begin{aligned}
\langle\varrho\rangle: \mathbb{R}_{*}^{+} \times \mathbb{R} & \rightarrow \mathbb{R}, \\
(t, z) & \mapsto R a_{x}(\varrho)\left(t, \omega_{1}, z\right)=\int_{\mathbb{R}^{N-1}} \varrho\left(t, z, x^{\prime \prime}\right) d x^{\prime \prime} .
\end{aligned}
$$

Theorem 1.2 asserts a $B V(\mathbb{R})$ control over $\langle\varrho\rangle(t, \cdot)$. We observe that such an information is certainly not easily accessible since the expression $\langle\varrho\rangle(\cdot)$ is visibly the solution of no (non linear) partial or integral differential equation. For example, taking the average of $\left(\mathcal{L}_{0}^{N}\right)$ with respect to the last variables, we only get:

$$
\partial_{t}\langle\varrho\rangle(t, z)+\partial_{z}\left\{\int_{\mathbb{R}^{N-1}} A_{1} \circ \varrho\left(t, z, x^{\prime \prime}\right) d x^{\prime \prime}\right\}=0,
$$

where the integration and the composition cannot be interchanged. This difficulty is inevitable when we restrict our attention to the space-time variables. On the other hand, it is removed by the kinetic interpretation:

$$
E(\varrho(t, z))=E(0)+\int_{\mathbb{R}} E^{\prime}(v) \chi_{\varrho(t, z)}(v) d v, \quad \forall E \in C^{1}(\mathbb{R}) .
$$

Indeed, it is now possible to pass under the integral sign:

$$
\begin{aligned}
& R a\left(\int_{\mathbb{R}} E^{\prime}(v) \chi_{\varrho(\cdot)}(v) d v\right)(\omega, z) \\
& =\int_{\mathbb{R}} E^{\prime}(v) R a_{x}\left(\chi_{\varrho(\cdot)}(\cdot)\right)(\omega, z, v) d v .
\end{aligned}
$$


From our perspective, property (2.21) is crucial in so far as improvement (1.7) stems from (2.21) exploited on the right of diagram $(\mathcal{D})$.

Now there is still an objection since in general the Radon transform and the kinetic action cannot be interchanged: $R a\left(\chi_{\varrho}\right)(\omega, z, v)$ is not equal to $\chi_{R a \varrho(\omega, z)}(v)$. This lack of commutation explains why the following statement:

$$
\begin{gathered}
\forall \omega \in \mathbb{S}^{N-1}, \forall E \in C^{2}(\mathbb{R}), \exists C(E) \in \mathbb{R}^{+} ; \forall t \in \mathbb{R}_{*}^{+}, \\
|E \circ \varrho(t, \cdot)|_{\mathcal{M}_{b, \omega}^{1}(\mathbb{R})} \leqslant C(E)\left|E \circ \varrho_{0}(\cdot)\right|_{\mathcal{M}_{b, \omega}^{1}(\mathbb{R})}
\end{gathered}
$$

is not directly available.

Such inequalities have not been demonstrated. Their validity (in particular when the flux $E(\cdot)$ is not convex) is not at all sure (even for the special choice $E \equiv \mathbb{I})$. These inequalities are typical of a propagation theorem where we have to estimate $\varrho(t, \cdot)$ knowing some information on $\varrho_{0}(\cdot)$. This link between the trace $\varrho(t, \cdot)$ and the Cauchy data $\varrho_{0}(\cdot)$ compels to work on the left of diagram $(\mathcal{D})$. Our scattering process allows precisely to remove this obligation.

The information (1.7) is distinct from (2.9). On the one hand, the condition $(\mathcal{N S})^{N}$ is not required. It means that the gap between $(\mathcal{L} \mathcal{D})^{N}$ and $(\mathcal{N S})^{N}$ is now filled. We see that the informations delivered are all the less precise as we approach the linear degenerate case (in particular, inequality $(1.7)$ is empty under $\left.(\mathcal{L D})^{N}-(2 \sim \mathrm{i})\right)$. On the other hand, the regularities given in (1.7) and (2.9) don't share the same nature. They cannot be compared (see Remark 5.1.1). In fact, the smoothing effect (1.7) can with difficulty lead to some Sobolev $W^{\tau, p}\left(\mathbb{R}^{N}\right)$ interpretation. However, it is easily expressed in the class $H^{\tau, \bar{\tau}}\left(\mathbb{R} \times \mathbb{R}^{N-1}\right)$ of Hörmander. In other words, it is possible to derivate a little in each direction $\omega$ certain well adjusted non linear expressions of $\varrho(\cdot)$ (that depend on the selected angle $\omega$ ) on condition that we accept to lose some derivatives perpendicular to $\omega$ :

Proposition 2.2.1 (Two-microlocal smoothing effect). - Fix any decomposition of $\mathbb{R}_{x}^{N}$ in a family of parallel hyperplanes with normal unit vector $\omega$ (see (0.1) for notations). We have:

$$
\begin{aligned}
& \forall A \in C^{2+n}\left(\mathbb{R} ; \mathbb{R}^{N}\right), \forall B \in C_{A}^{\omega}(\mathbb{R}), \exists C(A, B) \in \mathbb{R}^{+} ; \\
& \forall \tau \in]-\infty, \frac{1}{2}[, \forall \bar{\tau} \in]-\infty,-\frac{N+1}{2}\left[, \forall t \in \mathbb{R}_{*}^{+},\right.
\end{aligned}
$$




$$
\begin{aligned}
& \|B \circ \varrho(t, \cdot)\|_{H^{\tau, \bar{\tau}}\left(\mathbb{R}_{y^{\prime}}^{1} \times \mathbb{R}_{y^{\prime \prime}}^{N-1}\right)} \\
& \quad \leqslant C(A, B) \frac{(1+t)}{t}\left(\Theta\left(t, \varrho_{0}\right)+\left\|\varrho_{0}\right\|_{L^{1}\left(\mathbb{R}^{N}\right)}\right) .
\end{aligned}
$$

Remark 2.2.5. - This last statement is interesting only when $N \geqslant 2$. It thus cannot be deduced from (2.9) (or from Corollary 5.3.4). Indeed the parameter $\tau$ involved in inequality (2.9) is according to (2.12) far from the limit $1 / 2$ given at (2.22).

\section{THE SCATTERING OPERATOR}

\subsection{The strictly convex case $(N=1)$}

We point by this terminology the context $(1 \sim \mathrm{i})$ out. This situation is particularly favourable:

Proposition 3.1.1.- Assume $(\mathcal{V N} \mathcal{L})^{1}$. Then:

$$
\begin{aligned}
& \forall \varrho_{0} \in L_{c}^{\infty}(\mathbb{R}), \forall(t, s) \in \mathbb{R}^{+} \times[0, t], \exists \tilde{\varrho}(t, s, \cdot) \in L_{c}^{\infty}(\mathbb{R}) ; \\
& \operatorname{supp} \tilde{\varrho}(t, s, \cdot) \subset \operatorname{supp} \varrho_{0}+t V \text {, } \\
& \chi_{\varrho(t, x-(s-t) a(v))}(v)=\chi_{\varrho}(t, s, x)(v) .
\end{aligned}
$$

Furthermore, constraint (3.1) characterizes the physical solution $\varrho(\cdot)$ among all possible weak solutions of equation $\left(\mathcal{L}_{0}^{1}\right)$.

The contents of this statement is first illustrated by an example.

Example 3.1.1. - We consider the Bürger's law associated with initial data:

$$
\varrho_{0}(x):= \begin{cases}-1 & \text { if } x<0 \\ 1 & \text { if } x>0\end{cases}
$$

Two weak solutions $\varrho_{1}(\cdot)$ and $\varrho_{2}(\cdot)$ will be examined:

$$
\varrho_{1}(t, x)=\varrho_{0}(x), \quad \varrho_{2}(t, x):= \begin{cases}-1 & \text { if } x \leqslant-t, \\ \xi & \text { if } x=\xi t, \quad-1<\xi<1, \\ 1 & \text { if } t \leqslant x\end{cases}
$$

We draw in stippled line the corresponding backward characteristics (see Fig. 1).

On the left picture, the stippled straight lines are crossing each other at each intermediate time $s \in[0, t]$. It means that coming back with the operator $\Xi_{t}^{1}$ leads to the formation of a fold. In Brenier [4] and Perthame 


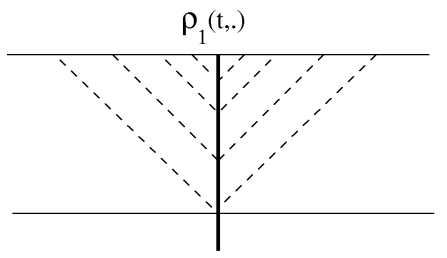

shock

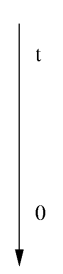

Fig. 1.

and Tadmor [24], this fold is corrected (and forbidden!) by the source term $\partial_{v} m(\cdot)$ of $\left(\mathcal{C}_{0}^{1}\right)$. In the absence of $\partial_{v} m(\cdot)$, when looking at $\left(\mathcal{T}_{t}^{1}\right)$ with $g_{t}(\cdot)$ that coincides with $\chi_{\varrho_{1}(t, \cdot)}(\cdot)$, a fold appears. Thus, we reject the function $\varrho_{1}(\cdot)$ since it fails to satisfy criterion (3.1).

On the right picture, the backward characteristics collide only at time $t=0$. It thus creates a centered compression wave, placed at $(0,0)$ which gives rise to a shock for negative times. But it does not matter since the process stops at $t=0$. As a result, the function $\varrho_{2}(\cdot)$ is acceptable with regard to criterion (3.1).

The solution sorted out by the condition (3.1) is the physical one.

It is well known that the evolution $\left(\mathcal{L}_{0}^{1}\right)$ is not reversible. With the help of the trace $\varrho(t, \cdot)$, it is not possible to recover the values of the solution $\varrho(\cdot)$ inside the cones of determination descended from the discontinuities. The scattering process completes these gaps by smoothing $\varrho(\cdot)$ in the places where the information is lost.

The non linear evolution which governs the formation of shocks is excluded to be replaced by a linear model. The graph of $g(\cdot)$ that is $\{(s, x, v)=(s, x, \tilde{\varrho}(t, s, x)) ;(s, x) \in[0, t] \times \mathbb{R}\}$ is hence formed by straight lines whose projections in $(s, x)$ coordinates get settled in a succession of fans (Fig. 2)

We now leave these formal considerations in order to present a rigorous demonstration:

Proof of Proposition 3.1.1. - Applied to initial data $\varrho_{0}(\cdot)$ in $B V(\mathbb{R})$, the criterion of reduction (3.1) is issued from two reasons. On the one hand, the well-known deterministic principle (evoked in Lax [15]) imposes that every point can be connected by a backward drawn characteristic to a point on the initial axis (for a convex law, characteristics may enter but may never emerge from a shock curve). On the other hand, the wave speed is monotone between the left and right values of $\varrho(t, \cdot)$ on 

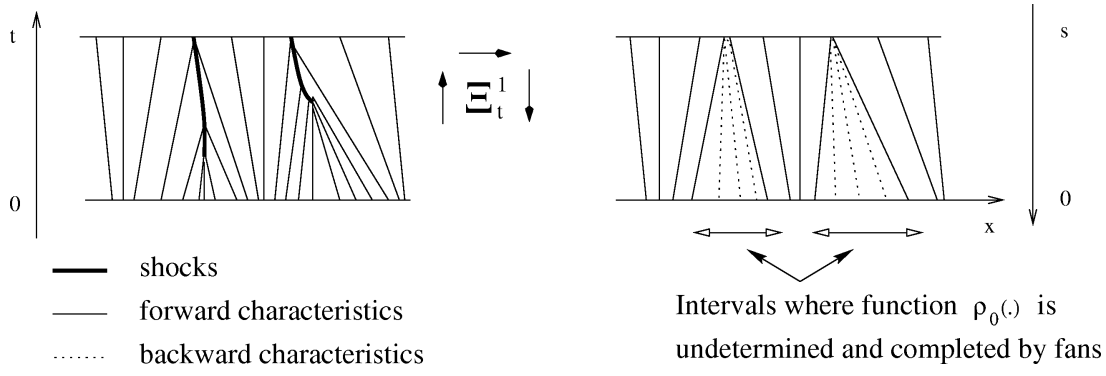

Fig. 2.

a shock which prevents the solution $g(s, \cdot)$ of being multivalued when coming back by the free transport equation.

In particular, the different characteristics passing through the extremal points of $\operatorname{supp} \varrho(t, \cdot)$ have null speed. It leads to inclusions $\operatorname{supp} \tilde{\varrho}(t, s, \cdot) \subset \operatorname{supp} \varrho(t, \cdot) \subset \operatorname{supp} \varrho_{0}+t V$ which justify (3.1).

We just have explained why the assertion of Proposition 3.1.1 holds for $\varrho_{0}(\cdot)$ in the space $B V(\mathbb{R})$. To cover the case $\varrho_{0}(\cdot)$ in $L^{\infty}(\mathbb{R})$, the reasoning rests on a smoothing argument. Let $\left(\varrho_{n}(\cdot)\right)_{n \in \mathbb{N}}$ be a sequence of Cauchy data subjected to:

$$
\lim _{n \rightarrow \infty}\left\|\varrho_{n}-\varrho_{0}\right\|_{L^{1}(\mathbb{R})}=0, \quad \varrho_{n} \in B V(\mathbb{R}), \forall n \in \mathbb{N}
$$

Let $\left(\bar{\varrho}_{n}(\cdot)\right)_{n \in \mathbb{N}}$ with $\bar{\varrho}_{n}(0, \cdot) \equiv \varrho_{n}$ be the sequence of corresponding solutions. By virtue of Proposition 3.1.1, for each $n \in \mathbb{N}$, we have:

$$
\exists \tilde{\varrho}_{n}(t, s, \cdot) \in L_{c}^{\infty}(\mathbb{R}) ; \quad \chi_{\varrho_{n}(t, x-(s-t) a(v))}(v)=\chi_{\tilde{\varrho}_{n}(t, s, x)}(v) .
$$

Moreover, a straightforward computation yields:

$$
\left\|\chi_{u}-\chi_{\bar{u}}\right\|_{L^{1}\left(\mathbb{R}^{2}\right)}=\|u-\bar{u}\|_{L^{1}(\mathbb{R})}, \quad \forall(u, \bar{u}) \in L_{c}^{\infty}(\mathbb{R})^{2} .
$$

Since the solution operator is a contraction in the space $L^{1}(\mathbb{R})$ (see Kružkov [14]), we get from (3.2):

$$
\lim _{n \longrightarrow \infty}\left\|\left(\bar{\varrho}_{n}-\varrho\right)(t, \cdot)\right\|_{L^{1}(\mathbb{R})} \leqslant \lim _{n \longrightarrow \infty}\left\|\varrho_{n}-\varrho_{0}\right\|_{L^{1}(\mathbb{R})}=0, \quad \forall t \in \mathbb{R}^{+} .
$$

The $L^{1}$ norms of solutions to $\left(\mathcal{T}_{t}^{N}\right)$ are unchanged. Hence:

$$
\lim _{n \longrightarrow \infty}\left\|\chi_{\varrho_{n}(t, \cdot-(s-t) a(\cdot))}(\cdot)-\chi_{\varrho(t, \cdot-(s-t) a(\cdot))}(\cdot)\right\|_{L^{1}\left(\mathbb{R}^{2}\right)}=0 .
$$


Combining (3.3), (3.4) and (3.5), we deduce that, for all $(t, s)$, the sequence $\left(\tilde{\varrho}_{n}(t, s, \cdot)\right)_{n \in \mathbb{N}}$ is of Cauchy type. Therefore it converges in $L^{1}(\mathbb{R})$ to a function $\tilde{\varrho}(t, s, \cdot)$ which is necessarily subjected to (3.1).

The entropy condition (in its geometrical formulation) requires that the different characteristics starting on either side of a discontinuity curve when continued in the direction of increasing $t$ intersect the line of discontinuity. By reversing the picture (see Example 3.1.1), we exactly recover (3.1). This observation shows that (3.1) characterizes physical solutions, as asserted.

As a direct consequence of Proposition 3.1.1, we infer Theorem 1.1 with $C(A)=2$. Applying Theorem 1.3, we then deduce (2.1).

Remark 3.1.1. - Inequality (1.4) can be refined. Let $x_{0}$ and $y_{0}$ be the endpoints determined by condition conv supp $\varrho_{0}=\left[x_{0}, y_{0}\right]$. It is well known (see Lax [15, p. 19]) that:

$$
\text { conv supp } \varrho(t, \cdot) \subset\left\{x ; x-a(0) t \in\left[x_{0}, y_{0}\right]+C(A) \sqrt{t}\right\} .
$$

It follows that:

$$
\Theta\left(t, \varrho_{0}\right) \leqslant 2 \min \left(\left|\operatorname{supp} \varrho_{0}+t V\right| ; y_{0}-x_{0}+2 C(A) \sqrt{t}\right) .
$$

Remark 3.1.2. - According to identities (3.1) and (1.2), we have (in the weak sense) for all positive time $t$ :

$$
t a^{\prime}(v) \partial_{x} g(t, x, v)=-\delta_{\tilde{\varrho}(t, 0, x-t a(v))}(v)+\delta_{\varrho(t, x)}(v) \leqslant \delta_{\varrho(t, x)}(v) .
$$

After integration with respect to $v$, this inequation yields the one-sidedLipshitz condition of Oleinik [22]:

$$
\partial_{x}(a \circ \varrho)(t, x) \leqslant 1 / t, \quad \forall x \in \mathbb{R}, \forall t \in \mathbb{R}_{*}^{+} .
$$

Since $\left(\mathcal{T}_{t}^{1}\right)$ is reversible, we notice by the way that $\tilde{\varrho}(t, 0, \cdot)$ is also in $B V(\mathbb{R})$ with a bound similar to (2.1). Observe also that control (1.4) is no more verified if the trace $\varrho(t, \cdot)$ is replaced by some application $\varrho_{t}(\cdot)$ that only satisfies at the time $t$ the compatibility condition on the jumps. Indeed, inequality (1.4) is a manifestation of the fact that the function $\varrho(\cdot)$ is an admissible solution on all the interval $[0, t]$ and not only on some subinterval of $[0, t]$.

The beautiful property (3.1) fails in the context $(1 \sim$ ii $)$. There is no reason for $g(s, \cdot)$ to remain a graph for every values of $s$ in $[0, t]$. This point is clear in view of the following counter-example: 


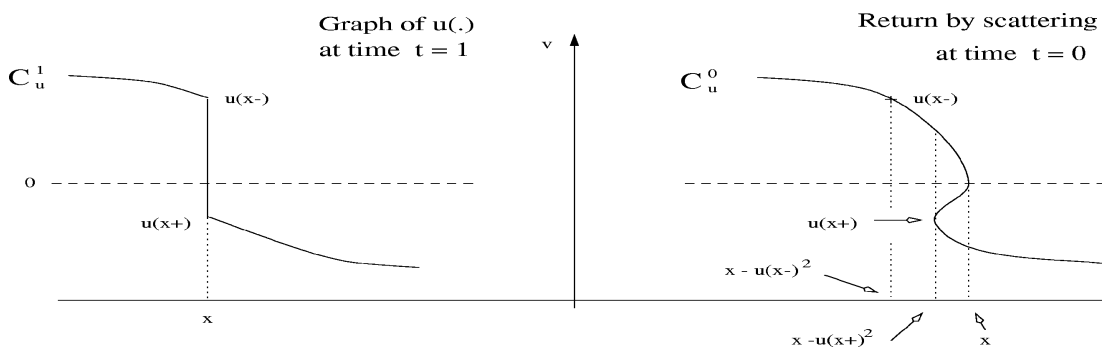

Fig. 3.

Counter-example 3.1. - Let us pick the mesh:

$$
x_{n}^{\bar{N}, \varepsilon}:=1+n \varepsilon-4^{-n}, \quad n \in\{0, \ldots, \bar{N}\},(\varepsilon, \bar{N}) \in \mathbb{R}_{*}^{+} \times \mathbb{N}_{*} .
$$

Now, consider the cubic law $\left(\mathcal{L}_{0}^{1}\right)$ associated to the initial data (that is suitably truncated for large values of $x$ ):

$$
\varrho_{0}^{\bar{N}, \varepsilon}(x):= \begin{cases}1 & \text { if } x<0 \\ (-1)^{n} 2^{-n} & \text { if } x_{n-1}^{\bar{N}, \varepsilon}<x<x_{n}^{\bar{N}, \varepsilon}, \quad n \in\{1, \ldots, \bar{N}\} \\ (-1)^{N} 2^{-N} & \text { if } x_{\bar{N}}^{\bar{N}, \varepsilon} \leqslant x\end{cases}
$$

The corresponding solution $\varrho^{\bar{N}, \varepsilon}(\cdot)$ is composed of a succession of $\bar{N}$ shocks that, for $n$ taken in $\{0, \ldots, \bar{N}-1\}$, issue from abscissa $x_{n}^{\bar{N}, \varepsilon}$ with slope $4^{-n}$ and reach at time 1 the position $1+n \varepsilon$. Letting parameter $\varepsilon$ tends to zero yields a compression wave placed in position $(t, x)=(1,1)$. Moreover:

$$
\lim _{\varepsilon \rightarrow 0}\left\|\left(\varrho^{\bar{N}, \varepsilon}-\varrho^{\bar{N}, 0}\right)(1, \cdot)\right\|_{L^{1}(\mathbb{R})}=0
$$

with:

$$
\varrho^{\bar{N}, 0}(1, x):= \begin{cases}1 & \text { if } x<1, \\ (-1)^{\bar{N}} 2^{-\bar{N}} & \text { if } 1<x .\end{cases}
$$

The graph of function $u(\cdot)$ is completed at the discontinuity points by a vertical segment:

$$
u_{s}(x):=(1-s) u(x-)+s u(x+), \quad s \in[0,1] .
$$

The coming back by the transport equation $\left(\mathcal{T}_{0}^{1}\right)$ of the curve

$$
C_{u}^{1}:=\left\{\mathbb{R}^{2} \ni(x, v)=\left(x, u_{s}(x)\right) ; x \in \mathbb{R}, s \in[0,1]\right\}
$$


is the path:

$$
C_{u}^{0}:=\left\{\mathbb{R}^{2} \ni(x, v)=\left(x-u_{s}(x)^{2}, u_{s}(x)\right) ; x \in \mathbb{R}, s \in[0,1]\right\} .
$$

The jumps $u(x-) / u(x+)$ that involve states with the same sign (i.e.: the product $u(x-) u(x+)$ is positive) are replaced by a fan according to Example 3.1.1.

The mixed jumps $u(x-) / u(x+)$ with switching sign (i.e.: the product $u(x-) u(x+)$ is negative) lead to the formation of a fold (double-valued solution) that at initial time is confined inside the interval of extremities $x-\min \left(u(x-)^{2}, u(x+)^{2}\right)$ and $x$.

Thus, the curve $C_{\varrho^{\bar{N}, \varepsilon}(t, \cdot)}^{0}$ is composed with $\bar{N}$ folds that come together and accumulate as parameter $\varepsilon$ tends to zero in the vicinity of abscissa $x=1$. Therefore:

$$
\exists \varepsilon \in \mathbb{R}_{*}^{+} ;\left\|\partial_{v} \Xi_{1}^{1}\left(\varrho_{0}^{\bar{N}, \varepsilon}\right)(1, \cdot)\right\|_{\mathcal{M}_{b}(\mathbb{R})}=4 \bar{N}
$$

The criterion (3.1) is clearly in contradiction with (3.8). It is not stable after passing to the limit since:

$$
\left\|\partial_{v}\left(\chi_{\varrho^{\bar{N}, 0}\left(1,1+v^{2}\right)}(v)\right)\right\|_{\mathcal{M}_{b}(\mathbb{R})} \leqslant 4 \neq 4 \bar{N}, \quad \forall \bar{N} \in \mathbb{N} \backslash\{0,1\} .
$$

Identity (3.8) shows that an uniform estimate of the type:

$$
\exists C \in \mathbb{R}^{+} ; \sup _{x \in \mathbb{R}^{N}}\left\|\partial_{v} \Xi_{t}^{N}\left(\varrho_{0}\right)(x, \cdot)\right\|_{\mathcal{M}_{b}(\mathbb{R})} \leqslant C, \forall \varrho_{0} \in L_{c}^{\infty}\left(\mathbb{R}^{N}\right)
$$

is not true. On the other hand, it does not contradict (1.4) since:

$$
\sup _{\bar{N} \in \mathbb{N}}\left|\Xi_{1}^{1}\left(\varrho_{0}^{\bar{N}, \varepsilon}\right)\right|_{\mathcal{M}_{b}^{0,1}\left(\mathbb{R}_{x}^{1} \times \mathbb{R}_{v}\right)} \leqslant 2 \sup _{\bar{N} \in \mathbb{N}}\left|\operatorname{supp} \varrho_{0}^{\bar{N}, \varepsilon}\right|+\sum_{n=1}^{\bar{N}} \frac{2}{4^{n+1}}<\infty
$$

Equality (3.1) implies (1.4) with $C(A)=2$. It is sufficient in order to deduce (1.4) but not necessary. Fortunately, in so far as it is violated by Counter-example 3.1.1 and, with greater reason, it is false in the extended situations $(1 \sim$ iii $),(2 \sim$ ii) $\ldots$. There, it has to be replaced by a more flexible constraint which incorporates the possibility for the acceleration $a^{\prime}(\cdot)$ to be equal to zero. In this direction, the bound (1.4) is adequate. Its relevance is borne out by the next paragraph. 


\subsection{The case with an inflection point $(N=1)$}

Our next project is to prove theorem 1.1 in the context $(1 \sim$ ii). For definiteness, we can suppose that $i_{A}=0$ and we can normalize the flux so that:

$$
\left\{\begin{array}{l}
A(0)=A^{\prime}(0)=A^{\prime \prime}(0)=0, \\
\left.\left.v A^{\prime \prime}(v)>0, \quad \forall|v| \in\right] 0, \varrho_{0}^{\infty}\right], \\
\left.\left.v_{A}:=\inf \left\{v A^{\prime}(v) / A(v) ;|v| \in\right] 0, \varrho_{0}^{\infty}\right]\right\}>1 .
\end{array}\right.
$$

Proof of Theorem 1.1. - It is sufficient to obtain the uniform control (1.4) for all BV solutions $\varrho(\cdot)$ corresponding to smooth initial data $\varrho_{0}(\cdot)$. Then, passing to the limit, we easily recover (1.4) if $\varrho_{0}(\cdot)$ is in $L_{c}^{\infty}(\mathbb{R})$.

When the flux $A(\cdot)$ has an inflection point, the extremal backward characteristics are no longer necessarily straight lines. Nevertheless, Dafermos [8] established that these characteristics are endowed with a special geometric structure. Under assumption $(\mathcal{I})^{1}$, the maximal backward characteristic $\zeta_{ \pm}(\cdot)$ through $(t, x \pm)$ is a convex Lipschitzian curve along which the speed $a \circ \varrho(s, \zeta(s)+)$ is continuous and decreasing. As a consequence, it is not sure that the value $\varrho(t, x)$ can be connected to some $\varrho_{0}(\tilde{x})$ (see Serre [26] - Chapter 2.5). This particularity makes the computations relative to the variation of $\varrho(\cdot)$ more complicated. This problem is faced in Zumbrun [30]. We present here another point of view that finally leads to more precise information.

Fix at the time $t>0$ some position $(t, x)$ where a discontinuity (of order zero) occurs. We denote by $\zeta_{-}(\cdot)$ and $\zeta_{+}(\cdot)$ the maximal backward characteristics issued respectively from the positions $(t, x-)$ and $(t, x+)$. According to this definition, we have:

$$
\begin{gathered}
\zeta_{ \pm}(t)=x, \quad \zeta_{-}(s)<\zeta_{+}(s), \quad \forall s \in[0, t[ \\
v_{+}(t):=\frac{d \zeta_{+}}{d s}(t)=a \circ \varrho(t, x+)<v_{-}(t):=\frac{d \zeta_{-}}{d s}(t)=a \circ \varrho(t, x-) . \\
0 \leqslant v_{ \pm}(s):=\frac{d \zeta_{ \pm}}{d s}(s)=a \circ \varrho\left(s, \zeta_{ \pm}(s)+\right), \quad \forall s \in[0, t[
\end{gathered}
$$

Let $|\mathcal{A}|$ be the area of the region $\mathcal{A}$ delimited by $\zeta_{-}(\cdot), \zeta_{+}(\cdot)$ and the initial axis:

$$
|\mathcal{A}|:=\int_{0}^{t}\left|\zeta_{+}(s)-\zeta_{-}(s)\right| d s
$$




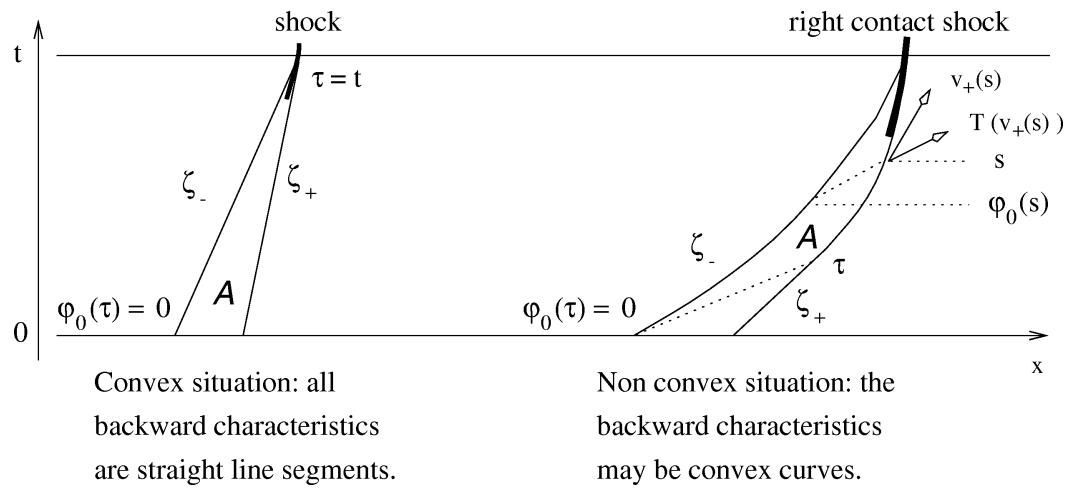

Fig. 4.

When both $\zeta_{-}(\cdot)$ and $\zeta_{+}(\cdot)$ are classical characteristics (it means: straight lines), we have:

$$
a \circ \varrho\left(s, \zeta_{ \pm}(s)+\right)=v_{ \pm}(t), \quad \forall s \in[0, t] .
$$

It follows that:

$$
|\mathcal{A}|=t^{2}\left(v_{-}(t)-v_{+}(t)\right) / 2 .
$$

When $\zeta_{-}(\cdot)$ (or $\left.\zeta_{+}(\cdot)\right)$ is incurved, identity (3.11) is no more valid. Nevertheless, we can substitute for (3.11) a convenient lower bound:

LEMMA 3.2.1. - In all cases:

$$
|\mathcal{A}| \geqslant \frac{\mu_{A}^{3} t^{2}}{432}\left(v_{-}(t)-v_{+}(t)\right), \quad 0<\mu_{A}:=1-\frac{1}{v_{A}}<1 .
$$

Proof of Lemma 3.2.1. - The key point is a good understanding of what happens when two states $\varrho_{-}$and $\varrho_{+}$are separated by a mixed shock (that is a shock with $\varrho_{-}$and $\varrho_{+}$on both sides of the inflection point; it means here: $\left.\varrho_{-} \varrho_{+}<0\right)($ Fig. 5).

Oleinik's condition is satisfied for a discontinuity $\varrho_{-} / \varrho_{+}$satisfying $\varrho_{+}-\varrho_{-}>0($ or $<0)$ when the chord that joins the points $\left(\varrho_{-}, A\left(\varrho_{-}\right)\right)$ and $\left(\varrho_{+}, A\left(\varrho_{+}\right)\right)$lies below (or above) the graph of $A(\cdot)$ between $\varrho_{-}$and $\varrho_{+}$. To simplify the discussion, we will only treat the case $\varrho_{-}>0$. The other situation $\varrho_{-}<0$ is completely similar.

Oleinik's condition means that the right state $\varrho_{+}$involved when constructing a mixed shock must be strictly confined inside $\varrho_{+}^{l}$ and 0 where the endpoint $\varrho_{+}^{l}$ (which corresponds to a right contact shock) is 


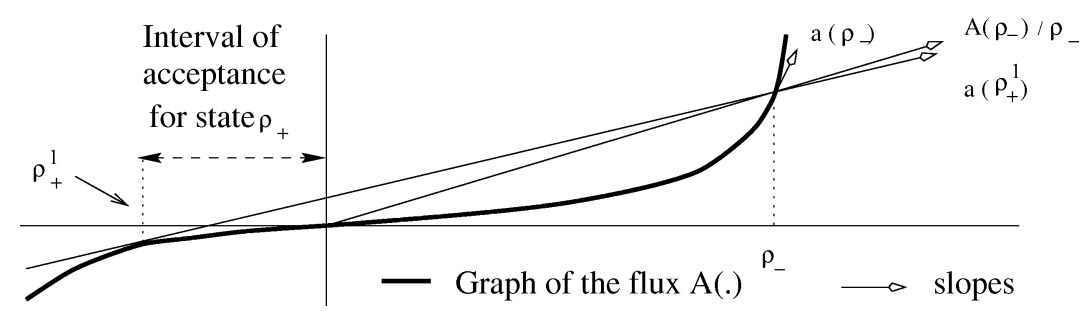

Fig. 5.

determined by the implicit relation:

$$
A\left(\varrho_{+}^{l}\right)-A\left(\varrho_{-}\right)-\left(\varrho_{+}^{l}-\varrho_{-}\right) a\left(\varrho_{+}^{l}\right)=0 .
$$

Given $\varrho_{-}>0$, Eq. (3.13) admits a unique negative solution. We define the transfer functions $T$ and $\bar{T}$ by the relations:

$$
a\left(\varrho_{-}\right)=T\left(a\left(\varrho_{+}^{l}\right)\right), \quad \varrho_{-}=\bar{T}\left(\varrho_{+}^{l}\right), \quad \forall \varrho_{-}>0 .
$$

In view of the preceding figure, we have for all admissible states $\varrho_{+}$in the interval $\left[\varrho_{+}^{l}, 0[\right.$ :

$$
\left.\left.0 \leqslant a\left(\varrho_{+}\right) \leqslant a\left(\varrho_{+}^{l}\right)<\frac{A\left(\varrho_{-}\right)}{\varrho_{-}}<a\left(\varrho_{-}\right), \quad \forall \varrho_{-} \in\right] 0, \varrho_{0}^{\infty}\right] .
$$

Remark 3.2.1. - For example, for the cubic law, we find:

$$
\left.\left.\left.\bar{T}(v)=-2 v, \forall|v| \in] 0, \varrho_{0}^{\infty}\right] \quad \text { and } \quad T(v)=4 v, \forall|v| \in\right] 0,\left(\varrho_{0}^{\infty}\right)^{2}\right] .
$$

The constraint $(\mathcal{I})^{1}$ is not at all restrictive since an inflection point is generically adjusted so that $(\mathcal{I})_{2}^{1}$ is true. Indeed, in general the property (2.19) is satisfied. It yields immediately:

$$
\lim _{\varrho \rightarrow 0} \frac{\varrho A^{\prime}(\varrho)}{A(\varrho)}=\lim _{\varrho \rightarrow 0} \frac{(m+1) ! A^{(m+1)}(0) \varrho^{m+1}}{m ! A^{(m+1)}(0) \varrho^{m+1}}=m+1 \geqslant 3 .
$$

Now, the application $\varrho \mapsto \varrho A^{\prime}(\varrho) / A(\varrho)$ is clearly continuous on $\left[-\varrho_{0}^{\infty}, 0[\cup] 0, \varrho_{0}^{\infty}\right]$. In view of $(3.14)$, it is strictly above 1 on this set. Combined with (3.15), it shows that number $v_{A}$ is strictly above 1 .

Condition $(\mathcal{I})^{1}$ also extends to certain fluxes whose derivatives up to any order are equal to zero at the origin (for instance consider the function that to $\varrho$ associates the expression $\left.\operatorname{sgn}(\varrho) \mathrm{e}^{-1 / \varrho^{2}}\right)$. However, there still 
exists some fluxes that have a degenerate inflection point (in the sense that $\left.\liminf _{\varrho \rightarrow 0} \varrho A^{\prime}(\varrho) / A(\varrho)=1\right)$. It does not mean that the associated estimate (1.4) is inexact. We will indeed not exploit all the margins that are available.

Combining (3.14) and $(\mathcal{I})^{1}$, we find:

$$
\begin{aligned}
a\left(\varrho_{-}\right) & \geqslant a\left(\varrho_{-}\right)-a\left(\varrho_{+}\right) \geqslant a\left(\varrho_{-}\right)\left(1-\frac{A\left(\varrho_{-}\right)}{\varrho_{-} a\left(\varrho_{-}\right)}\right) \\
& \geqslant \mu_{A} a\left(\varrho_{-}\right) \geqslant \mu_{A} a\left(\varrho_{+}\right), \quad \forall \varrho_{+} \in\left[\varrho_{+}^{l}, 0[.\right.
\end{aligned}
$$

Inequality (3.16) is important. It means that the difference between $a\left(\varrho_{-}\right)$and $a\left(\varrho_{+}\right)$cannot be fixed arbitrarily. This property distinguishes mixed shocks from usual shocks (with $\varrho_{-} \varrho_{+} \geqslant 0$ ) for which the number $a\left(\varrho_{-}\right)-a\left(\varrho_{+}\right)$can be taken as small as wanted. As a consequence, we can refine (3.12):

LEMMA 3.2.2. - Suppose that $(t, x)$ is the position of a right contact shock. Then the corresponding area $\mathcal{A}$ is bounded above by:

$$
|\mathcal{A}| \geqslant \mu_{A}^{3} t^{2} v_{-}(t) / 216 .
$$

Proof of Lemma 3.2.2. - By hypothesis:

$$
v_{-}(t)=a \circ \varrho(t, x-)=T\left(v_{+}(t)\right)=T(a \circ \varrho(t, x+)) .
$$

For $s \in[\tau, t]$, the straight line issued from the position $\left(s, \zeta_{+}(s)\right.$ with slope $T\left(v_{+}(s)\right)$ intersects the curve $\zeta_{-}(\cdot)$ at the point $\left(\varphi_{0}(s), \zeta_{-} \circ \varphi_{0}(s)\right)$ (see Fig. 4). It is necessarily tangent to the curve $\zeta_{-}(\cdot)$. For $s \in[0, \tau]$, it reaches the initial axis. If $\zeta_{-}(\cdot)$ is a straight segment, we have $\tau=t$. Otherwise, we get $0<\tau<t$ and by convention $\varphi_{0}(\tau)=0$.

The path $\zeta_{-}(\cdot)$ can obviously be recovered by the family of its tangent lines:

$$
\left\{(r, x) ; x=r T\left(v_{+}(s)\right)+\zeta_{+}(s)-s T\left(v_{+}(s)\right\}_{s \in[\tau, t]} .\right.
$$

The bigger $T$, the more these lines are inclined. This fact implies that the area $\mathcal{A}$ is increasing with $T$ :

$$
\left.\left.T_{1}(v) \geqslant T_{2}(v), \forall v \in\right] 0, \varrho_{0}^{\infty}\right] \Rightarrow\left|\mathcal{A}_{T_{1}}\right| \geqslant\left|\mathcal{A}_{T_{2}}\right| .
$$

Since by $(\mathcal{I})^{1}$ and (3.14) the authorized transfer functions are subject to $T(v) \geqslant v_{A} v$, we only have to work with the special choice $T(v)=v_{A} v$. 


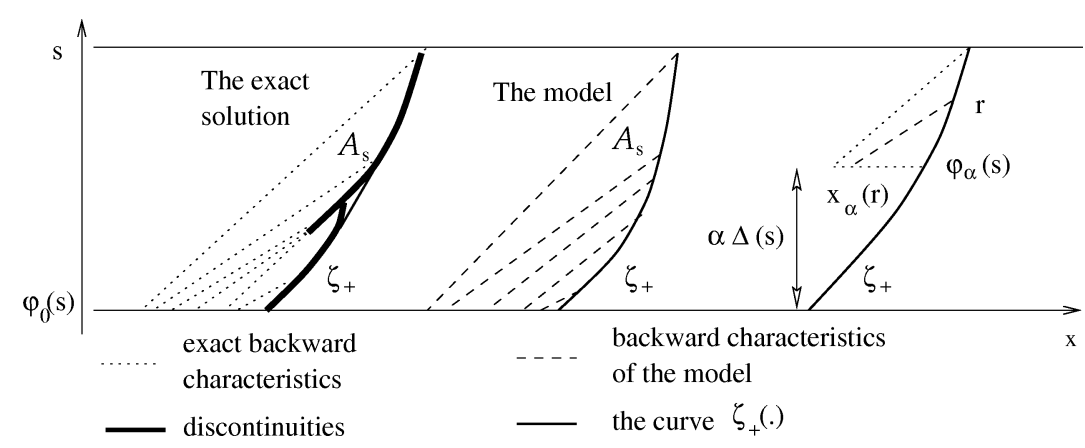

Fig. 6.

This remark will notably simplify the analysis. An easy computation leads to an explicit formula for the gap:

$$
\left.\left.\Delta(s):=s-\varphi_{0}(s)=-\mu_{A} v_{+}(s) / v_{+}^{\prime}(s)>0, \quad v_{+}^{\prime}(s)<0, \quad \forall s \in\right] \tau, t\right] .
$$

Let us fix $\alpha \in[0,1]$ and set $\varphi_{\alpha}(s):=\varphi_{0}(s)+\alpha \Delta(s)$. At this stage, we need some intermediate result:

LEMMA 3.2.3. -

$$
\begin{gathered}
\zeta_{+} \circ \varphi_{\alpha}(s)-\zeta_{+}(s)+(1-\alpha) v_{A} \Delta(s) v_{+}(s) \\
\geqslant \alpha(1-\alpha)\left(v_{A}-1\right) \Delta(s) v_{+}(s), \\
\forall(\alpha, s) \in[0,1] \times[\tau, t] .
\end{gathered}
$$

Proof of Lemma 3.2.3. - Let us consider the domain $\mathcal{A}_{s}$ delimited on the left by the inclined segment $\left\{\left(\varphi_{\alpha}(s), \zeta_{+}(s)-v_{A}(1-\alpha) \Delta(s) v_{+}(s)\right)\right.$; $\alpha \in[0,1]\}$, on the right by the piece of curve $\left\{\zeta_{+}(r) ; r \in\left[\varphi_{0}(s), s\right]\right\}$ and below by the horizontal line $\left\{\left(\varphi_{0}(s), x\right) ; \zeta_{-}(s) \leqslant x \leqslant \zeta_{+}(s)\right\}$. We clearly have the inclusions: $\mathcal{A}_{s} \subset \mathcal{A}, \forall s \in[\tau, t]$ (Fig. 6).

Now, the formation of a right contact shock comes necessarily from the presence of a rarefaction wave on the left side (that possibly radiates out of some other contact discontinuity). Exploiting this particularity, we can forget the exact history of the formation of the curve $\zeta_{+}(\cdot)$.

We can replace inside $\mathcal{A}_{s}$ the exact solution by a function (still denoted by $\varrho(\cdot))$ that is constant along segments. The slope of a segment issued from a point $\left(s, \zeta_{+}(s)\right)$ on $\zeta_{+}(\cdot)$ must coincide with $v_{A} v_{+}(s)$ and the function $\varrho(\cdot)$ is there taken to be constant equal to $\bar{T}\left(\varrho\left(s, \zeta_{+}(s)+\right)\right.$. The entropy condition implies that these segments cannot cross one other. This manipulation allows to deal with backward characteristics which 
are partly right contact shocks and partly (straight line) characteristics. In fact, it induces a modification only between regions delimited by backward characteristics issued from endpoints of straight segments included in $\zeta_{+}(\cdot)$ (where the true characteristics are replaced by a family of straight parallel lines; see the preceding picture).

We perform the analysis at the level of the aforesaid model, with $s$ fixed. We introduce the position:

$$
x_{\alpha}(r):=\zeta_{+}(r)-v_{A}\left(r-\varphi_{\alpha}(s)\right) v_{+}(r), \quad r \in\left[\varphi_{\alpha}(s), s\right]
$$

and the width:

$$
l_{\alpha}(r):=\zeta_{+} \circ \varphi_{\alpha}(s)-x_{\alpha}(r), \quad r \in\left[\varphi_{\alpha}(s), s\right]
$$

The curve $\zeta_{+}(\cdot)$ is completely determined by the speeds of propagation of the incoming rarefaction wave. We introduce the family of functions $\left(H_{\alpha}(\cdot)\right)_{\alpha \in[0,1]}$ defined by the relation:

$$
H_{\alpha}\left(x_{\alpha}(r)\right)=\bar{T}\left(\varrho\left(r, \zeta_{+}(r)+\right)\right), \quad r \in\left[\varphi_{\alpha}(s), s\right] .
$$

With this convention, the application $H_{\alpha}(\cdot)$ is increasing on the interval $\left[\zeta_{-} \circ \varphi_{\alpha}(s), \zeta_{+} \circ \varphi_{\alpha}(s)\right]$. In fact, when $\left.\left.\alpha \in\right] 0,1\right]$, we recover a more subtle estimate that expresses the regularizing effect due to spreading of rarefaction waves. To see this point, just consider the positions:

$$
z(y):=y+\alpha \Delta(s) a \circ H_{0}(y), \quad y=x_{0}(r), \quad r \in\left[\varphi_{0}(s), s\right],
$$

and observe that we have by construction:

$$
H_{\alpha}(z(y))=H_{0}(y), \quad H_{\alpha}^{\prime}(z) \geqslant 0 .
$$

The derivation of (3.23) with respect to $y$ ensures that:

$$
0 \leqslant \alpha \Delta(s) H_{\alpha}^{\prime}(z(y)) a^{\prime} \circ H_{0}(y)=1-\left[H_{\alpha}^{\prime}(z) / H_{0}^{\prime}(y)\right] \leqslant 1 .
$$

Using (3.23) again, we deduce:

$$
0 \leqslant a^{\prime} \circ H_{\alpha}(z) H_{\alpha}^{\prime}(z) \leqslant 1 /[\alpha \Delta(s)] .
$$

We plug (3.22) inside (3.20) and use definition (3.10) to find:

$$
l_{\alpha}(r)=\zeta_{+} \circ \varphi_{\alpha}(s)-\zeta_{+}(r)+\left(r-\varphi_{\alpha}(s)\right) a \circ H_{\alpha} \circ x_{\alpha}(r)
$$


After derivation with respect to $r$, it yields:

$l_{\alpha}^{\prime}(r)=\left(v_{A}-1\right) v_{+}(r) /\left[1+\left(r-\varphi_{\alpha}(s)\right) a^{\prime} \circ H_{\alpha} \circ x_{\alpha}(r) H_{\alpha}^{\prime} \circ x_{\alpha}(r)\right]$

By virtue of (3.24) and the decreasing of $v_{+}(\cdot)$, we infer that:

$$
l_{\alpha}^{\prime}(r) \geqslant \alpha\left(v_{A}-1\right) v_{+}(s), \quad \forall r \in\left[\varphi_{\alpha}(s), s\right] .
$$

Hence, after integration from $r=\varphi_{\alpha}(s)$ to $r=s$ :

$$
l_{\alpha}(s) \geqslant\left(v_{A}-1\right) \alpha(1-\alpha) \Delta(s) v_{+}(s), \quad \forall \alpha \in[0,1]
$$

which is exactly (3.19).

As a direct consequence of (3.19), we find:

$$
\left|\mathcal{A}_{s}\right|=\Delta(s) \int_{0}^{1} l_{\alpha}(s) d \alpha \geqslant\left(v_{A}-1\right) v_{+}(s) \Delta(s)^{2} / 6
$$

To obtain Lemma 3.2.2, we argue by contradiction. We suppose that:

$$
\left(v_{A}-1\right) v_{+}(s) \Delta(s)^{2} \leqslant \mu_{A}^{3} t^{2} v_{-}(t) / 36, \quad \forall s \in[\tau, t],
$$

and show that it is impossible. Therefore:

$$
\exists \bar{s} \in[\tau, t] ; \quad\left(v_{A}-1\right) v_{+}(\bar{s}) \Delta(\bar{s})^{2} \geqslant \mu_{A}^{3} t^{2} v_{-}(t) / 36
$$

which guarantees (3.17) since by (3.25) and (3.27):

$$
|\mathcal{A}| \geqslant\left|\mathcal{A}_{\bar{s}}\right| \geqslant\left(v_{A}-1\right) v_{+}(\bar{s}) \Delta(\bar{s})^{2} / 6 \geqslant \mu_{A}^{3} t^{2} v_{-}(t) / 216
$$

We then interpret (3.26) as a differential inequality

$$
\frac{d}{d s}\left\{v_{+}(s)^{-1 / 2}\right\} \geqslant \frac{3}{t \sqrt{v_{+}(t)}}, \quad \forall s \in[\tau, t],
$$

which requires after integration:

$$
0 \leqslant \frac{3(t-s)}{t \sqrt{v_{+}(t)}} \leqslant \frac{1}{\sqrt{v_{+}(t)}}-\frac{1}{\sqrt{v_{+}(s)}} \leqslant \frac{1}{\sqrt{v_{+}(t)}}, \quad \forall s \in[\tau, t] .
$$



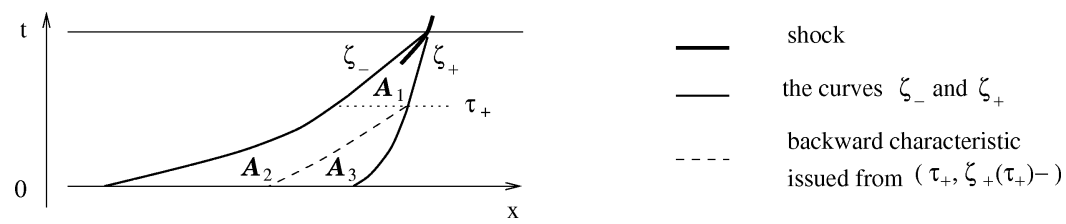

Fig. 7.

Assumption (3.26) written with $s=\tau$ gives:

$$
\Delta(\tau) \leqslant \frac{\mu_{A} t}{6}\left(\frac{\mu_{A} v_{-}(t)}{\left(v_{A}-1\right) v_{+}(\tau)}\right)^{1 / 2}=\frac{\mu_{A} t}{6}\left(\frac{v_{-}(t)}{v_{-}(\tau)}\right)^{1 / 2} \leqslant \frac{\mu_{A} t}{6} .
$$

Inequality (3.28) is consistent only if $t-\tau \leqslant t / 3$. Combined with (3.29), it yields:

$$
0<t=t-\tau+\Delta(\tau) \leqslant \frac{t}{3}+\frac{\mu_{A} t}{6} \leqslant \frac{t}{2}
$$

which is the expected contradiction.

Finally, we turn to the proof of Lemma 3.2.1. According to inequalities (3.11) and (3.17), cases consisting in a classical shock and in a right contact shock are already gained. Thus, we can restrict our attention to the other situations. Now, for $s \in\left[\tau_{ \pm}, t\right]$ with $0<\tau_{-}<t$ or $0<\tau_{+}<t$, the backward characteristic $\zeta_{ \pm}(\cdot)$ is a straight line segment whereas it becomes strictly convex at time $\tau_{ \pm}$.

We cut up the surface $\mathcal{A}$ in three parts $\mathcal{A}_{1}, \mathcal{A}_{2}$ and $\mathcal{A}_{3}$ defined on Fig. 7. Clearly:

$$
\begin{gathered}
|\mathcal{A}|=\left|\mathcal{A}_{1}\right|+\left|\mathcal{A}_{2}\right|+\left|\mathcal{A}_{3}\right| . \\
\left|\mathcal{A}_{1}\right| \geqslant\left(t-\tau_{+}\right)^{2}\left(v_{-}(t)-v_{+}(t)\right) / 2 .
\end{gathered}
$$

Since it appears a right contact shock at the position $\left(\tau_{+}, \zeta_{+}\left(\tau_{+}\right)\right)$, we can apply lemma 3.2.2 to the area $\mathcal{A}_{3}$ to get:

$$
\left|\mathcal{A}_{3}\right| \geqslant \mu_{A}^{3} \tau_{+}^{2} T\left(v_{+}\left(\tau_{+}\right)\right) / 216 \geqslant \mu_{A}^{3} \tau_{+}^{2} v_{-}(t) / 216 .
$$

Combining (3.30), (3.31) and (3.32), we find (3.12). The proof of Lemma 3.2.1 is complete. 


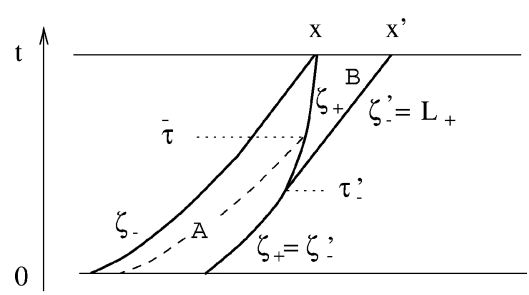

the right backward characteristic issued from $\left(t, x^{\prime}\right)$ becomes incurved at time $\tau$,

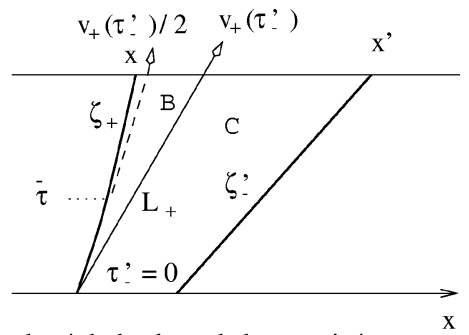

the right backward characteristic issued from $\left(t, x^{\prime}\right)$ is still a straight line segment

Fig. 8.

We now study the passage between two successive discontinuities. The notations are as before. We just add a prime ' when dealing with the shock on the right (and not to mark a derivation) (Fig. 8).

We introduce the straight line segment:

$$
L_{+}:=\left\{(s, y) ; \tau_{-}^{\prime} \leqslant s \leqslant t, y=\zeta_{+}\left(\tau_{-}^{\prime}\right)+v_{+}\left(\tau_{-}^{\prime}\right)\left(s-\tau_{-}^{\prime}\right)\right\} .
$$

We denote by $B$ the surface delimited above by the horizontal line $s=t$, on the left by the curve $\zeta_{+}(\cdot)$ and on the right by $L_{+}$. We designate by $C$ the surface bordered by $s=t, s=0, L_{+}$and $\zeta_{-}^{\prime}(\cdot)$. With this convention, the domain $C$ is absent if $\tau_{-}^{\prime}>0$.

LEMMA 3.2.4. -

$$
\max (|B| ;|\mathcal{A}|) \geqslant \mu_{A}^{3} t^{2} v_{+}\left(\tau_{-}^{\prime}\right) / 2000, \quad|C| \geqslant t\left(\zeta_{-}^{\prime}(0)-\zeta_{+}(0)\right) / 2
$$

Proof of Lemma 3.2.4. - Let us consider the intermediate time:

$$
\bar{\tau}:=\min \left\{s \in\left[\tau_{-}^{\prime}, t\right] ; 2 v_{+}(s) \geqslant v_{+}\left(\tau_{-}^{\prime}\right)\right\}
$$

If $\bar{\tau} \leqslant t / 2$, we estimate $|B|$ :

$$
|B| \geqslant(t-\bar{\tau})^{2}\left(v_{+}\left(\tau_{-}^{\prime}\right)-v_{+}(\bar{\tau})\right) / 2 \geqslant t^{2} v_{+}\left(\tau_{-}^{\prime}\right) / 16 \text {. }
$$

If $\bar{\tau} \geqslant t / 2$, we refer to Lemma 3.2.2 applied to the position $\left(\bar{\tau}, \zeta_{+}(\bar{\tau})\right)$. Since the corresponding area is obviously less than $|\mathcal{A}|$, it yields:

$$
|\mathcal{A}| \geqslant \mu_{A}^{3} \bar{\tau}^{2} T\left(v_{+}(\bar{\tau})\right) / 216 \geqslant \mu_{A}^{3} t^{2} v_{+}\left(\tau_{-}^{\prime}\right) / 2000 .
$$


If $\zeta_{-}^{\prime}(0)>\zeta_{+}(0)$, the maximal backward characteristic $\zeta_{-}^{\prime}(\cdot)$ is necessarily a straight line that does not cross $L_{+}$. Therefore, the minoration given for $|C|$ is immediate.

Finally, we implement the preceding lemmas in order to get (1.4). By approximation, it suffices to obtain (1.4) for a solution $\varrho(\cdot)$ that has a finite number of discontinuities. We mark their positions at time $t$ :

$$
-\infty<c^{0}(t) \leqslant c^{1}(t)<\cdots<c^{J}(t) \leqslant c^{J+1}(t)<+\infty .
$$

In this interpretation, $c^{0}(t)$ and $c^{J+1}(t)$ denote the endpoints of the convex hull of supp $\varrho(t, \cdot)$. By convention, a symbol is indexed by $j$ when dealing with the discontinuity $c^{j}(t)$. The strip $[0, t] \times \mathbb{R}$ is partitioned into $J+1$ regions $\mathcal{R}^{1}, \ldots, \mathcal{R}^{J+1}$ bordered according to:

$$
\mathcal{R}^{j}:=\left\{(s, x) ; 0 \leqslant s \leqslant t, \zeta_{-}^{j}(s) \leqslant x \leqslant \zeta_{-}^{j+1}(s)\right\}, \quad 0 \leqslant j \leqslant J .
$$

The complete graph (see (3.7)) of the restriction $\bar{\varrho}^{j}(t, \cdot)$ of the trace $\varrho^{j}(t, \cdot)$ on the interval $\left[c^{j}(t), c^{j+1}(t)\right.$ [ brings a contribution $\Theta_{j}\left(t, \varrho_{0}\right)$. More precisely:

$$
\Theta_{j}\left(t, \varrho_{0}\right):=\left\|\partial_{v}\left\{\chi_{\varrho^{j}(t, x+t a(v))}(v)\right\}\right\|_{\mathcal{M}_{b}\left(\mathbb{R}^{2}\right)} .
$$

Our aim is to control each $\Theta_{j}\left(t, \varrho_{0}\right)$. From this perspective, we classify the domains $\mathcal{R}^{j}$ according to the nature of the shock involved at the position $\left(t, c^{j}(t)\right)$.

a) Usual shock $\left(\varrho_{-} \varrho_{+} \geqslant 0\right)$. In the convex framework, it is the only situation that occurs. A short computation yields:

$$
\begin{aligned}
\Theta_{j}\left(t, \varrho_{0}\right) / 2= & t\left(v_{-}^{j}(t)-v_{+}^{j}(t)\right)+\zeta_{-}^{j+1}(0)-\zeta_{+}^{j}(0) \\
& +c^{j}(t)-t v_{+}^{j}(t)-\zeta_{+}^{j}\left(\tau_{-}^{j+1}\right)+\tau_{-}^{j+1} v_{+}^{j}\left(\tau_{-}^{j+1}\right) \\
\leqslant & t\left(v_{-}^{j}(t)-v_{+}^{j}(t)\right)+t v_{+}^{j}\left(\tau_{-}^{j+1}\right)+\zeta_{-}^{j+1}(0)-\zeta_{+}^{j}(0) .
\end{aligned}
$$

b) Mixed shock $\left(\varrho_{-} \varrho_{+}<0\right)$. Discontinuities with switching sign lead to the formation of a fold (see the picture of Counter-example 3.1.1) whose width evaluated at $s=0$ is equal to $t v_{+}^{j}(t)$. We have to incorporate this new contribution in the preceding expression:

$$
\Theta_{j}\left(t, \varrho_{0}\right) / 2 \leqslant t v_{-}^{j}(t)+t v_{+}^{j}\left(\tau_{-}^{j+1}\right)+\zeta_{-}^{j+1}(0)-\zeta_{+}^{j}(0)
$$


Combining Lemmas 3.2.1, 3.2.4 and (3.16), we find:

$$
t \Theta_{j}\left(t, \varrho_{0}\right) \leqslant 8000\left(\left|\mathcal{A}^{j}\right|+\left|B^{j}\right|+\left|C^{j}\right|\right) / \mu_{A}^{4}, \quad \forall j \in\{0, \ldots, J\} .
$$

We only need to sum the contributions $\Theta_{j}\left(t, \varrho_{0}\right)$ to get:

$$
t \Theta\left(t, \varrho_{0}\right)=t \sum_{j=0}^{J} \Theta_{j}\left(t, \varrho_{0}\right) \leqslant \frac{8000}{\mu_{A}^{4}} \int_{0}^{t}|\operatorname{supp} \varrho(s, \cdot)| d s .
$$

Thereby, the bound (1.4) becomes an easy consequence of the finite speed of propagation.

Remark 3.2.2. - When the flux $A(\cdot)$ has several inflection points, it becomes more difficult to unravel the intricacies of the shock set (see Dafermos [8]). Inequalities (3.16) are no more true. In particular, a discontinuity could be simultaneously a right and left contact shock. At present, we are not able to take into account these non local effects. However, since locally the situation is acquired, the bound (1.4) is probably true whatever the number of inflection points is.

\subsection{The multidimensional case $(N \geqslant 2)$}

We use in this paragraph the notations of Vol'pert [29]: $\Gamma(\varrho)$ is the set of points of jump for $\varrho(\cdot)$; v denotes a unit vector normal to $\Gamma(\varrho) ; \Delta \varrho(t, x)=\left(l_{\nu} \varrho-l_{-\nu} \varrho\right)(t, x)$ is the jump of $\varrho(\cdot)$ at $(t, x) \ldots$ We introduce the distribution:

$$
D(t, \cdot):=\operatorname{div}(a \circ \varrho)(t, \cdot)=\sum_{i=1}^{N} \partial_{x_{i}}\left(a_{i} \circ \varrho\right)(t, \cdot) \in \mathcal{S}^{\prime}\left(\mathbb{R}_{x}^{N}\right),
$$

and the expressions:

$$
B_{i}(x, v):=\int_{-\infty}^{v} a_{i}^{\prime}(r) b(x, r) d r, \quad \forall(x, v) \in \mathbb{R}_{x}^{N} \times \mathbb{R}_{v}, i \in[1, N] .
$$

LEMMA 3.3.1. - The mass of the divergence of the wave speed is controlled by $\Theta\left(t, \varrho_{0}\right)$ whatever the flux $A(\cdot)$ is:

$$
t\|D(t, \cdot)\|_{\mathcal{M}_{b}\left(\mathbb{R}^{N}\right)} \leqslant \Theta\left(t, \varrho_{0}\right), \quad \forall\left(t, \varrho_{0}\right) \in \mathbb{R}_{*}^{+} \times L_{c}^{\infty}\left(\mathbb{R}^{N}\right) .
$$


Assume that $A(\cdot)$ is admissible. Then, the converse is true. There exists some positive constant $C(A)$ such that:

$$
\Theta\left(t, \varrho_{0}\right) \leqslant 2|\operatorname{supp} \varrho(t, \cdot)|+C(A) t\|D(t, \cdot)\|_{\mathcal{M}_{b}\left(\mathbb{R}^{N}\right)} .
$$

Proof of Lemma 3.3.1. - By definition, number $\Theta\left(t, \varrho_{0}\right)$ is the same as:

$$
\begin{gathered}
\Theta\left(t, \varrho_{0}\right)=\sup \left\{\left|\int_{\mathbb{R}^{N}} \int_{\mathbb{R}} g_{0}(x, v) \partial_{v}[b(x+\operatorname{ta}(v), v)] d x d v\right| ;\right. \\
\left.b \in C_{c}^{1}\left(\mathbb{R}_{x}^{N} \times \mathbb{R}_{v}\right),\|b\|_{C^{0}\left(\mathbb{R}_{x}^{N} \times \mathbb{R}_{v}\right)} \leqslant 1\right\} .
\end{gathered}
$$

The different integral terms which occur in this supremum are interpreted by adding a well adjusted null contribution:

$$
\begin{aligned}
\iint & g_{0}(x, v) \partial_{v}[b(x+t a(v), v)] d x d v \\
= & \iint g(t, x, v) \partial_{v} b(x, v) d x d v \\
& +t \iint g(t, x, v) a^{\prime}(v) \cdot \nabla_{x} b(x, v) d x d v \\
& -t \int \operatorname{div}\left(\int g(t, x, v) b(x, v) a^{\prime}(v) d v\right) d x \\
= & \int b(x, \varrho(t, x)) d x-\int b(x, 0) d x \\
& +t \sum_{i=1}^{N} \int\left\{\left(\partial_{x_{i}} B_{i}\right)(x, \varrho(t, x))-\partial_{x_{i}}\left[B_{i}(x, \varrho(t, x))\right]\right\} d x \\
= & \int b(x, \varrho(t, x)) d x-\int b(x, 0) d x \\
& -t \int_{\mathbb{R}^{N} \backslash \Gamma(\varrho)} b(x, \varrho(t, x)) D(t, x) d x \\
& -t \int_{\Gamma(\varrho)} e_{v}(t, x) \triangle \varrho(t, x) \mathbb{H}_{N-1}(d x),
\end{aligned}
$$

where we have used the functional superposition:

$$
e_{v}(t, x):=b \hat{a}_{v}^{\prime}(x, \varrho(t, x))=\int_{0}^{1}\left(b a_{v}^{\prime}\right)\left(x, l_{-\nu} \varrho(t, x)+r \Delta \varrho(t, x)\right) d r
$$


Identity (3.35) written with $b(\cdot)$ independent of $v$ yields:

$$
\iint g_{0}(x, v) \partial_{v}[b(x+t a(v))] d x d v=-t \int D(t, x) b(x) d x .
$$

Taking the supremum on $b(\cdot)$ in (3.36), we obtain the first part (3.33) of Lemma 3.3.1. Then, we deduce from (3.35) that:

$$
\begin{aligned}
& \left|\int_{\mathbb{R}^{N}} \int_{\mathbb{R}} g_{0}(x, v) \partial_{v}[b(x+\operatorname{ta}(v), v)] d x d v\right| \\
& \leqslant 2|\operatorname{supp} \varrho(t, \cdot)|+t \int_{\mathbb{R}^{N} \backslash \Gamma(\varrho)}|D(t, x)| d x \\
& \quad+t \int_{\Gamma(\varrho)}\left|e_{v}(t, x) \Delta \varrho(t, x)\right| \mathbb{H}_{N-1}(d x) .
\end{aligned}
$$

The functional calculus of Vol'pert [29] gives:

$$
\begin{aligned}
\|D(t, \cdot)\|_{\mathcal{M}_{b}\left(\mathbb{R}^{N}\right)} & =\int_{\mathbb{R}^{N} \backslash \Gamma(\varrho)}|D(t, x)| d x \\
& +\int_{\Gamma(\varrho)}\left|\triangle\left(a_{v} \circ \varrho\right)(t, x)\right| \mathbb{H}_{N-1}(d x) .
\end{aligned}
$$

Thereby, to get (3.34), we only have to show that:

$$
\left|e_{\nu}(t, x) \triangle \varrho(t, x)\right| \leqslant C(A)\left|\triangle\left(a_{v} \circ \varrho\right)(t, x)\right|, \quad(t, x) \in \Gamma(\varrho) .
$$

Let us consider any jump $\varrho_{-} / \varrho_{+}$placed at a position $(t, x)$ on the set $\Gamma(\varrho)$. If the polarized flux $A_{v}(\cdot)$ do satisfy $(\mathcal{L D})^{1}$, inequality $(3.37)$ is trivial since both terms are null. If $A_{v}(\cdot)$ is genuine non linear $(\mathcal{V N} \mathcal{L})^{1}$ or if $\varrho_{-}$and $\varrho_{+}$are in the same side of the inflection point, the function $a_{v}^{\prime}(\cdot)$ keeps a constant sign on the interval separating $\varrho_{-}$and $\varrho_{+}$. It follows that:

$$
\left|e_{v}(t, x) \triangle \varrho(t, x)\right| \leqslant\left|\triangle\left(a_{v} \circ \varrho\right)(t, x)\right|, \quad(t, x) \in \Gamma(\varrho) .
$$

Suppose now that the discontinuity $\varrho_{-} / \varrho_{+}$is a mixed shock. By condition $(\mathcal{I})_{1}^{1}$, we have:

$$
\begin{aligned}
\left|e_{v}(t, x) \triangle \varrho(t, x)\right| \leqslant & \left|a_{v}\left(l_{\nu} \varrho(t, x)\right)-a_{v}\left(i_{A_{v}}\right)\right| \\
& +\left|a_{v}\left(l_{-v} \varrho(t, x)\right)-a_{v}\left(i_{A_{v}}\right)\right| .
\end{aligned}
$$


Combining (3.14) and (3.16), we deduce the majoration:

$$
\left|e_{v}(t, x) \triangle \varrho(t, x)\right| \leqslant 2\left|\triangle\left(a_{\nu} \circ \varrho\right)(t, x)\right| / \mu_{A},
$$

which completes the proof of (3.37).

At this stage, Theorem 1.2 becomes a corollary of the following result:

Proposition 3.3.1. - Assume that the flux $A(\cdot)$ is admissible and that the hypothesis $(\mathcal{H})$ (given $p .41)$ is true. Then, the divergence $D(t, \cdot)$ belongs to the space $\mathcal{M}_{b}\left(\mathbb{R}^{N}\right)$. More precisely:

$$
t\|D(t, \cdot)\|_{\mathcal{M}_{b}\left(\mathbb{R}^{N}\right)} \leqslant C(|\operatorname{supp} \varrho(t, \cdot)|), \quad \forall t \in \mathbb{R}_{*}^{+} .
$$

Proof of Proposition 3.3.1. - The demonstration is decomposed in three steps (1), (2) and (3). It is first given in a sketchy (but very significant) way. Technical details are then furnished.

It is sufficient to get an uniform control on quantities $D(t, \cdot)$ which correspond to solutions issued from $C^{\infty}$ initial data. Now, such solutions are generically smooth (say $C^{2}$ ) outside a set of jumps $\Gamma(\varrho)$ which is a countable union of surfaces of discontinuities. The distribution $D(t, \cdot)$ can always be split into:

$$
\mathcal{M}_{b}\left(\mathbb{R}^{N}\right) \ni D(t, \cdot)=D^{+}(t, \cdot)-D^{-}(t, \cdot), \quad D^{ \pm}(t, \cdot) \geqslant 0 .
$$

$\mapsto(1)$ : Since $D(t, \cdot)$ has zero mean, we have:

$$
\|D(t, \cdot)\|_{\mathcal{M}_{b}\left(\mathbb{R}^{N}\right)}=2\left\|D^{+}(t, \cdot)\right\|_{\mathcal{M}_{b}\left(\mathbb{R}^{N}\right)} .
$$

Therefore, to get (3.38), we can concentrate on $D^{+}(t, \cdot)$.

$\mapsto(2)$ : Oleinik's condition implies that $\triangle\left(a_{v} \circ \varrho\right)(t, x)$ is negative on the set of jump $\Gamma(\varrho)$. It follows that for all subset $\Gamma \subset \Gamma(\varrho)$, we have:

$$
\int_{\Gamma} D(t, x) \mathbb{H}_{N-1}(d x)=\int_{\Gamma} \triangle\left(a_{\nu} \circ \varrho\right)(t, x) \mathbb{H}_{N-1}(d x) \leqslant 0 .
$$

It means that the singularities (of order zero) of the solution $\varrho(\cdot)$ contribute only to $D^{-}(t, \cdot)$. They are not seen at the level of $D^{+}(t, \cdot)$. This fact has an important consequence. When carrying out the ana- lysis at the level of $D^{+}(\cdot)$, we work in the domain $\mathbb{R}^{N} \backslash \Gamma(\varrho)$ where the function $D^{+}(\cdot)$ is sufficiently regular to use differential calculus. 
$\mapsto(3)$ : It follows that $D^{+}(\cdot)$ does satisfy the constraint:

$$
\partial_{t} D^{+}(t, x)+\sum_{i=1}^{N} a_{i} \circ \varrho(t, x) \partial_{x_{i}} D^{+}(t, x)+D^{+}(t, x)^{2}=0
$$

which is valid in each connected component of the set:

$$
\Upsilon:=\left\{(t, x) ; D^{+}(t, x)>0\right\} \subset\left(\mathbb{R}^{N} \backslash \Gamma(\varrho)\right) .
$$

The identity (3.41) indicates that $D^{+}(t, x)$ is controlled by $1 / t$ once the backward characteristic issued from $(t, x)$ is well defined and included in $\Upsilon$ on the whole interval $[0, t]$. Such an estimate is in agreement with (3.38).

The rigorous implementation of steps (1), (2) and (3) comes up against technical difficulties. On the one hand, the relevant information (3.38) does not involve an $L^{\infty}$-norm but an $L^{1}$-norm. This first objection is overcome by introducing a convenient functional $f_{\mu}(\cdot)$ (defined next page). On the other hand, the separation between $\Gamma(\varrho)$ and $\Upsilon$ may be thin. It can reduce to zero. Moreover, near a point $(t, x)$ placed on a contact surface, the expression $D^{+}(t, y)$ tends to $+\infty$ when the position $(t, y)$ converges towards $(t, x)$ on one side of $\Gamma(\varrho)$. This second disadvantage is compensated by condition $(\mathcal{I})_{2}^{1}$ which implies the (strict and crucial !) inequality $\triangle\left(a_{v} \circ \varrho\right)(t, x)<0$ on $\Gamma(\varrho)$. It means that the jump is the dominant singularity at $(t, x)$. It follows that the sets $\Gamma(\varrho)$ and $\Upsilon$ are effectively separated after regularizing the solution $\varrho(\cdot)$. We propose now a (not exclusive) way to tackle these two difficulties. Let $\varrho_{\mu}(\cdot)$ be the parabolic approximation of $\varrho(\cdot)$ defined by:

$$
\begin{aligned}
\partial_{t} \varrho_{\mu}(t, x)+\sum_{i=1}^{N} \partial_{x_{i}}\left(A_{i} \circ \varrho_{\mu}\right)(t, x) & =\mu \Delta \varrho_{\mu}(t, x) \\
& =\mu \sum_{i=1}^{N} \partial_{x_{i}}^{2} \varrho_{\mu}(t, x) .
\end{aligned}
$$

Let us consider the divergence of the corresponding wave speed:

$$
D_{\mu}(t, \cdot):=\operatorname{div}\left(a \circ \varrho_{\mu}\right)(t, \cdot)=\sum_{i=1}^{N} \partial_{x_{i}}\left(a_{i} \circ \varrho_{\mu}\right)(t, \cdot) \in C_{c}^{\infty}\left(\mathbb{R}_{x}^{N}\right) .
$$

We denote by $D_{\mu}^{+}(t, \cdot)$ and $D_{\mu}^{-}(t, \cdot)$ respectively the positive part and the negative part of function $D_{\mu}(t, \cdot)$. An easy computation shows that 
the quantity $D_{\mu}(t, \cdot)$ is subjected to:

$$
\partial_{t} D_{\mu}(t, x)+\sum_{i=1}^{N} \partial_{x_{i}}\left[a_{i} \circ \varrho_{\mu} D_{\mu}\right](t, x)=\mu \mathcal{E} r_{\mu}(t, x),
$$

with:

$$
\begin{aligned}
\mathcal{E} r_{\mu}(t, x) & :=\sum_{i=1}^{N} \partial_{x_{i}}\left[a_{i}^{\prime} \circ \varrho_{\mu} \Delta \varrho_{\mu}\right](t, x) \\
& =\Delta D_{\mu}(t, x)-\sum_{i=1}^{N} \partial_{x_{i}}\left[a_{i}^{\prime \prime} \circ \varrho_{\mu}\left|\nabla \varrho_{\mu}\right|^{2}\right](t, x) .
\end{aligned}
$$

We introduce the (unique) positive scalar $C_{\mu}(t)$ that realizes:

$$
\int_{\mathcal{D}_{\mu}(t)} D_{\mu}(t, x) d x=C_{\mu}(t), \quad \mathcal{D}_{\mu}(t):=\left\{x \in \mathbb{R}^{N} ; D_{\mu}(t, x) \geqslant C_{\mu}(t)\right\} .
$$

With this convention, we have necessarily:

$$
\begin{gathered}
D_{\mu}(t, x)=C_{\mu}(t), \quad \forall x \in \partial \mathcal{D}_{\mu}(t), \\
0 \leqslant D_{\mu}^{+}(t, x) \leqslant C_{\mu}(t), \quad \forall x \in \mathbb{R}^{N} \backslash \mathcal{D}_{\mu}(t), \\
\left\|D_{\mu}^{+}(t, \cdot)\right\|_{L^{1}\left(\mathbb{R}^{N)}\right)}=\int_{\mathcal{D}_{\mu}(t)} D_{\mu}^{+}(t, x) d x+\int_{\mathbb{R}^{N} \backslash \mathcal{D}_{\mu}(t)} D_{\mu}^{+}(t, x) d x .
\end{gathered}
$$

We deduce from (3.40), (3.45) and (3.46) the following majorations:

$$
2 C_{\mu}(t) \leqslant\left\|D_{\mu}(t, \cdot)\right\|_{L^{1}\left(\mathbb{R}^{N}\right)} \leqslant 2\left(1+\left|\operatorname{supp} \varrho_{\mu}(t, \cdot)\right|\right) C_{\mu}(t) .
$$

We set $f_{\mu}(t):=\mathrm{e}^{-\left|\mathcal{D}_{\mu}(t)\right|} C_{\mu}(t)$ and compute its derivative with respect to time $t$. We use (3.42), (3.44) and Stoke's formula to find:

$$
\begin{aligned}
\mathrm{e}^{\left|\mathcal{D}_{\mu}(t)\right|} \frac{d}{d t} f_{\mu}(t) & \\
\quad= & \int_{\mathcal{D}_{\mu}(t)} \partial_{t} D_{\mu}(t, x) d x \\
& =-\sum_{i=1}^{N} \int_{\mathcal{D}_{\mu}(t)} \partial_{x_{i}}\left[a_{i} \circ \varrho_{\mu} D_{\mu}\right](t, x) d x+\mu \int_{\mathcal{D}_{\mu}(t)} \mathcal{E} r_{\mu}(t, x) d x
\end{aligned}
$$


C. CHEVERRY / Ann. Inst. Henri Poincaré 17 (2000) 413-472

$$
\begin{aligned}
& =-\int_{\partial \mathcal{D}_{\mu}(t)}\left[D_{\mu} a_{v} \circ \varrho_{\mu}\right](t, x) \mathbb{H}_{N-1}(d x)+\oint_{\mu}(t) \\
& =-C_{\mu}(t) \int_{\mathcal{D}_{\mu}(t)} D_{\mu}(t, x) d x+\oint_{\mu}(t)=-C_{\mu}(t)^{2}+\oint_{\mu}(t) \\
& \leqslant-\mathrm{e}^{2\left|\mathcal{D}_{\mu}(t)\right|} f_{\mu}(t)^{2}+\oint_{\mu}(t) .
\end{aligned}
$$

In (3.48), the undefined term is computed by using the two forms of definition (3.43). We find:

$$
\begin{aligned}
\oint_{\mu}(t) & :=\mu \int_{\partial \mathcal{D}_{\mu}(t)}\left[a_{\nu}^{\prime} \circ \varrho_{\mu} \Delta \varrho_{\mu}\right](t, x) \mathbb{H}_{N-1}(d x) \\
& =\mu \int_{\partial \mathcal{D}_{\mu}(t)}\left[\partial_{\nu} D_{\mu}-a_{\nu}^{\prime \prime} \circ \varrho_{\mu}\left|\nabla \varrho_{\mu}\right|^{2}\right](t, x) \mathbb{H}_{N-1}(d x), \\
\partial_{\nu} & :=\sum_{i=1}^{N} v_{i} \partial_{x_{i}} .
\end{aligned}
$$

Since the sequence $\left\{D_{\mu}(t, \cdot)\right\}_{\mu>0}$ converges weakly in $L^{1}\left(\mathbb{R}^{N}\right)$ towards $D(t, \cdot)$, the majoration (3.38) becomes a consequence of:

$$
\begin{aligned}
\|D(t, \cdot)\|_{\mathcal{M}_{b}\left(\mathbb{R}^{N}\right)} & \leqslant \limsup _{\mu \rightarrow 0}\left\|D_{\mu}(t, \cdot)\right\|_{L^{1}\left(\mathbb{R}^{N}\right)} \\
& \leqslant C(|\operatorname{supp} \varrho(t, \cdot)|)<\infty .
\end{aligned}
$$

To verify the uniform control (3.49) by applying (3.47) and Gronvall's lemma to the differential inequality (3.48), it clearly suffices to suppose that the following hypothesis $(\mathcal{H})$ is valid for all $\left(t, \varrho_{0}\right)$ in $\mathbb{R}_{*}^{+} \times C_{c}^{\infty}\left(\mathbb{R}^{N}\right)$ :

$$
\lim _{\mu \rightarrow 0} \mu \int_{0}^{t} \oint_{\mu}(s) d s \leqslant C(|\operatorname{supp} \varrho(t, \cdot)|)\left(1+f_{\mu}(t)\right) .
$$

First notice that $\partial_{\nu} D_{\mu}(t, \cdot)$ is negative on the boundary $\partial \mathcal{D}_{\mu}(t)$. Indeed, function $D_{\mu}(t, \cdot)$ is by construction decreasing from the interior to the exterior of the domain $\mathcal{D}_{\mu}(t)$. Thereby, for a quadratic (multidimensional) law, we are sure that $\oint_{\mu}(t) \leqslant 0$. In this simple case, $(\mathcal{H})$ is obvious.

Finally, we explain why assumption $(\mathcal{H})$ should be true in the general situation. In fact we need an additional argument to compensate the (possibly positive) quadratic contribution $-a_{\nu}^{\prime \prime} \circ \varrho_{\mu}(t, \cdot)\left|\nabla \varrho_{\mu}(t, \cdot)\right|^{2}$ by 
the negative term $\partial_{\nu} D_{\mu}(t, \cdot)$. At this stage the argument is formal (for $N=1$, it corresponds to the hard core of the demonstration given in Section 3.2). By condition $(\mathcal{I})_{2}^{1}$, it is natural to expect that:

$$
\lim _{\mu \rightarrow 0} D_{\mu}(t, x)=-\infty, \quad \forall(t, x) \in \Gamma(\varrho) .
$$

In other words, the contribution due to points on $\Gamma(\varrho)$ should be asymptotically negligible:

$$
\bigcap_{\mu>0}\left(\mathcal{D}_{\mu}(t) \cap \Gamma(\varrho)\right)=\emptyset .
$$

Now, out of $\Gamma(\varrho)+\varepsilon$ with $\varepsilon>0$ fixed, the scalar $\left|\nabla \varrho_{\mu}(t, \cdot)\right|$ is uniformly bounded whereas the product $\mu\left|\nabla \varrho_{\mu}(t, \cdot)\right|$ should be well controlled or less (in the sense of inequality $(\mathcal{H})$ ) than $-\mu \partial_{\nu} D_{\mu}(t, \cdot)$ in the remainder set $(\Gamma(\varrho)+\varepsilon) \backslash \Gamma(\varrho)$. These observations and Section 3.2 indicate why hypothesis $(\mathcal{H})$ is grounded.

\section{TRANSFER OF REGULARITY BY AVERAGING}

We have to deal with a basic principle that can be observed on equation $\left(\mathcal{T}_{0}^{1}\right)$. The solution of $\left(\mathcal{T}_{0}^{1}\right)$ with initial condition $g_{0}(\cdot)$ is given by an explicit formula which leaves the $L^{1}\left(\mathbb{R}_{x} \times \mathbb{R}_{v}\right)$-norm unchanged:

$$
g(s, x, v)=g_{0}(x-s a(v), v), \quad\|g(s, \cdot)\|_{L^{1}\left(\mathbb{R}^{2}\right)}=\left\|g_{0}\right\|_{L^{1}\left(\mathbb{R}^{2}\right)} .
$$

We focus our attention on some special class of Cauchy data that inherit suitable features with respect to our purpose:

DEFINITION 4.1. - We say that a function $g_{0}(\cdot)$ in $L^{\infty}\left(\mathbb{R}^{2}\right)$ is well prepared up to the order $(m, n) \in \mathbb{N}^{2}$ for the flux $A(\cdot)$ if it decomposes according to a product

$$
g_{0}(x, v)= \begin{cases}b_{0}(v) \bar{g}_{0}(x, v), & \text { if } n=0, \\ b_{n}(v) a^{\prime}(v)^{2 n-1} \bar{g}_{n}(x, v), & \text { if } n>0,\end{cases}
$$

whose constituents are subjected to:

$$
b_{n} \in C_{c}^{n}(\mathbb{R}), \quad \bar{g}_{n} \in \mathcal{M}_{b}^{m, n}\left(\mathbb{R}_{x} \times \mathbb{R}_{v}\right) .
$$

Remark 4.1. - Of course, if function $g_{0}(\cdot)$ is well prepared up to the order $(m, n)$, it is so also for the other pairs $(\bar{m}, \bar{n})$ satisfying $0 \leqslant \bar{m} \leqslant m$ and $0 \leqslant \bar{n} \leqslant n$. 
The next proposition reveals the property that we have in mind:

Proposition 4.1. - We suppose that the initial data $g_{0}(\cdot)$ has support contained in the strip $\mathbb{R} \times\left[-\varrho_{0}^{\infty}, \varrho_{0}^{\infty}\right]$ and that it is well prepared up to the order $(m, n)$ for the flux $A(\cdot)$. At each positive time $s$, we then have the bound:

$$
\left|\int_{\mathbb{R}} g(s, \cdot, v) d v\right|_{\mathcal{M}_{b}^{m+n}(\mathbb{R})} \leqslant \frac{C\left(n, a, b_{n}\right)}{s^{n}}\left\|\bar{g}_{n}\right\|_{\mathcal{M}_{b}^{m, n}\left(\mathbb{R}_{x} \times \mathbb{R}_{v}\right)} .
$$

Remark 4.2. - The constant involved in (4.4) can be computed:

$$
\begin{gathered}
C\left(n, a, b_{n}\right) \leqslant C(n)\left(\|a\|_{C^{n}\left(\left[-\varrho_{0}^{\infty}, \varrho_{0}^{\infty}\right]\right)}+\left\|b_{n}\right\|_{C^{n}\left(\left[-\varrho_{0}^{\infty}, \varrho_{0}^{\infty}\right]\right)}\right) . \\
C\left(k, a, b_{k}\right)=\left\|b_{k}\right\|_{C^{k}\left(\left[-\varrho_{0}^{\infty}, \varrho_{0}^{\infty}\right]\right)}, \quad k \in\{0,1\} .
\end{gathered}
$$

Proof of Proposition 4.1. - By density, we just have to prove all the assertions with the symbol $\mathcal{M}_{b}$ replaced by $L^{1}$. By virtue of (4.1) and (4.2), we have:

$$
g(s, x, v)=b_{n}(v) a^{\prime}(v)^{2 n-1} \bar{g}_{n}(x-s a(v), v) .
$$

We mention now some formula that will be used repeatedly in the sequel. By expanding the derivative inside the brackets in the following integral:

$$
0=\int_{\mathbb{R}} \partial_{v}\left\{b_{n}(v) a^{\prime}(v)^{l-1}\left(\partial_{x}^{\bar{l}} \bar{g}_{n}\right)(x-s a(v), v)\right\} d v, \quad(l, \bar{l}) \in \mathbb{N}_{*} \times \mathbb{N},
$$

we obtain the identity:

$$
\begin{aligned}
& s \int_{\mathbb{R}} b_{n}(v) a^{\prime}(v)^{l}\left(\partial_{x}^{\bar{l}+1} \bar{g}_{n}\right)(x-s a(v), v) d v \\
& =\int_{\mathbb{R}}\left(\partial_{v} b_{n}\right)(v) a^{\prime}(v)^{l-1}\left(\partial_{x}^{\bar{l}} \bar{g}_{n}\right)(x-s a(v), v) d v \\
& \quad+\int_{\mathbb{R}} b_{n}(v) \partial_{v}\left[a^{\prime}(\cdot)^{l-1}\right](v)\left(\partial_{x}^{\bar{l}} \bar{g}_{n}\right)(x-s a(v), v) d v \\
& \quad+\int_{\mathbb{R}} b_{n}(v) a^{\prime}(v)^{l-1}\left(\partial_{x}^{\bar{l}} \partial_{v} \bar{g}_{n}\right)(x-s a(v), v) d v .
\end{aligned}
$$


The left part of inequality (4.4) can be written:

$$
\begin{aligned}
\int_{\mathbb{R}}\left|\partial_{x}^{m+n}\left\{\int_{\mathbb{R}} g(s, x, v) d v\right\}\right| d x \\
=\int_{\mathbb{R}}\left|\partial_{x}^{n}\left\{\int_{\mathbb{R}} b_{n}(v) a^{\prime}(v)^{2 n-1}\left(\partial_{x}^{m} \bar{g}_{n}\right)(x-s a(v), v) d v\right\}\right| d x .
\end{aligned}
$$

It is therefore enough to consider the pairs $\{(0, n)\}_{n \in \mathbb{N}}$. We first discuss the case $n=0$ and $n=1$ apart. When $n=0$, we have to deal with:

$$
\int_{\mathbb{R}}\left|\int_{\mathbb{R}} b_{0}(v) \bar{g}_{0}(x-s a(v), v) d v\right| d x .
$$

We pass the absolute value $|\cdot|$ under the sign sum and then proceed to the change of variables $\Psi:(x, v) \mapsto(x-s a(v), v)$ to obtain (4.4) with the constant given in (4.6).

When $n=1$, we exploit (4.8) with the choice $(l, \bar{l})=(1,0)$. Interpreting the first integral in terms of our solution $g(\cdot)$, we obtain:

$$
\begin{aligned}
s \partial_{x}\left\{\int_{\mathbb{R}} g(s, z, v) d v\right\}= & \int_{\mathbb{R}}\left(\partial_{v} b_{1}\right)(v) \bar{g}_{1}(x-s a(v), v) d v \\
& +\int_{\mathbb{R}} b_{1}(v)\left(\partial_{v} \bar{g}_{1}\right)(x-s a(v), v) d v .
\end{aligned}
$$

The integrands on the right hand side of (4.10) are evaluated as before, by making use of $\Psi$. This yields again (4.4) with the constant indicated at (4.6). The key point here is the special structure of $g_{0}(\cdot)$ which allows to transfer the derivative in $x$ towards a derivative in $v$.

Following the same principle, we now proceed by induction on the index $n$.

The first step $n=1$ has already been achieved. We assume now that the result (4.4) is true up to the order $n-1$ with $n-1>0$. We are looking at what occurs at level $n$. Using again (4.8) but this time with the choice $(l, \bar{l})=(2 n-1, n-1)$, we find:

$$
s \partial_{x}^{n}\left\{\int_{\mathbb{R}} g(s, x, v) d v\right\}=\sum_{k=1}^{3} \partial_{x}^{n-1}\left\{\int_{\mathbb{R}} g^{k}(s, x, v) d v\right\} .
$$


Above, functions $g^{k}$ correspond to the resolution of $\left(\mathcal{T}_{0}^{1}\right)$ where the initial data are decomposed according to:

$$
g^{k}(x, v):=b_{n-1}^{k}(v) a^{\prime}(v)^{2(n-1)-1} \bar{g}_{n-1}^{k}(x, v), \quad k \in\{1,2,3\},
$$

with:

$$
\begin{gathered}
b_{n-1}^{1}(v):=\left(\partial_{v} b_{n}\right)(v) a^{\prime}(v), \quad \bar{g}_{n-1}^{1}(z, v)=\bar{g}_{n}(z, v) . \\
b_{n-1}^{2}(v):=(2 n-2) b_{n}(v) a^{\prime \prime}(v), \quad \bar{g}_{n-1}^{2}(z, v)=\bar{g}_{n}(z, v) . \\
b_{n-1}^{3}(v):=b_{n}(v) a^{\prime}(v), \quad \bar{g}_{n-1}^{3}(z, v):=\left(\partial_{v} \bar{g}_{n}\right)(z, v) .
\end{gathered}
$$

We see that:

$$
\begin{aligned}
& b_{n-1}^{k} \in C_{c}^{n-1}(\mathbb{R}), \quad \forall k \in\{1,2,3\} . \\
& \left\|\bar{g}_{n-1}^{k}\right\|_{\left(L^{1}\right)^{0, n-1}\left(\mathbb{R}^{2}\right)} \leqslant\left\|\bar{g}_{n}\right\|_{\left(L^{1}\right)^{0, n}\left(\mathbb{R}^{2}\right)}, \quad \forall k \in\{1,2,3\} .
\end{aligned}
$$

Obviously, the three functions $g^{k}(\cdot)$ are well prepared up to the order $(0, n-1)$ for the flux $A(\cdot)$. According to the induction hypothesis, each term on the right hand of identity (4.11) can be analyzed. This is how we obtain inequality (4.4).

Remark 4.3. - Equation $\left(\mathcal{T}_{0}^{1}\right)$ does not involve any source term. There is an extended literature on the case with second member. Mostly [9,11, $12]$, the regularity is evaluated in terms of both $t$ and $x$ variables. There is also an optimal version due to Bouchut and Desvilettes [2] where $t$ is fixed. Our approach differs from these previous standpoints in that we exploit special features on the initial data. This is the reason why we can go further.

\section{APPLICATIONS}

The previous general considerations are now applied in order to obtain concrete results.

\subsection{Proof of Theorem 1.3}

Proof. - The results obtained in Section 4 incorporate the case $N \geqslant 2$ by appealing to the Radon transform. Indeed the function

$$
\tilde{g}(s, \omega, z, v)=\tilde{T}_{0, \omega}^{N}\left(s, g_{0}\right)(z, v):=\left(R a_{x} g\right)(s, \omega, z, v)
$$


issued from the solution $g(\cdot)$ of $\left(\mathcal{T}_{0}^{N}\right)$ is subjected to the following one dimensional transport equation:

$$
\left(\tilde{\mathcal{T}}_{0, \omega}^{N}\right):=\left\{\begin{array}{l}
\partial_{s} \tilde{g}(s, \omega, z, v)+\omega \cdot a(v) \partial_{z} \tilde{g}(s, \omega, z, v)=0 \\
\tilde{g}(0, \omega, z, v)=\tilde{g}_{0}(\omega, z, v)
\end{array}\right.
$$

where $\omega \in \mathbb{S}^{N-1}$ is simply a parameter.

An easy computation shows that:

$$
\sup _{\omega \in \mathbb{S}^{N-1}}\left|\tilde{g}_{0}(\omega, \cdot)\right|_{\mathcal{M}_{b}^{0,1}\left(\mathbb{R}_{z} \times \mathbb{R}_{v}\right)} \leqslant|g(0, \cdot)|_{\mathcal{M}_{b}^{0,1}\left(\mathbb{R}_{x}^{N} \times \mathbb{R}_{v}\right)}=\Theta\left(t, \varrho_{0}\right)
$$

In order to get rid of the assumptions required in Definition 4.1, we fix some $b(\cdot)$ in $C_{c}^{1}(\mathbb{R})$ (with associated $B(\cdot)$ according to formula (1.6)) and multiply $\tilde{g}_{0}(\cdot)$ by $b(v) \omega \cdot a^{\prime}(v)$. The resulting expression:

$$
b(v) \omega \cdot a^{\prime}(v) \tilde{g}_{0}(\omega, z, v), \quad b \in C^{1}(\mathbb{R}), \omega \in \mathbb{S}^{N-1}
$$

is then well prepared up to the order $(0,1)$. From Proposition 4.1, follows:

$$
\begin{aligned}
& \left|\int_{\mathbb{R}} b(v) \omega \cdot a^{\prime}(v) \tilde{g}(t, \omega, \cdot, v) d v\right|_{\mathcal{M}_{b}^{1}(\mathbb{R})} \\
& \quad=\left|R a_{x}\left(\int_{\mathbb{R}} b(v) \omega \cdot a^{\prime}(v) \chi_{\varrho(t, \cdot)}(v) d v\right)(\omega, \cdot)\right|_{\mathcal{M}_{b}^{1}(\mathbb{R})} \\
& \quad=|B \circ \varrho(t, \cdot)|_{\mathcal{M}_{b, \omega}^{1}(\mathbb{R})} \leqslant\|b\|_{C^{1}\left(\left[-\varrho_{0}^{\infty}, \varrho_{0}^{\infty}\right]\right)}\left(\Theta\left(t, \varrho_{0}\right)+\left\|\varrho_{0}\right\|_{L^{1}\left(\mathbb{R}^{N}\right)}\right) / t .
\end{aligned}
$$

We thus have verified the assertion of Theorem 1.3.

Remark 5.1.1. - Let us interpret more carefully the information:

$$
\exists \omega \in \mathbb{S}^{N-1} ; \quad|u|_{\mathcal{M}_{b, \omega}^{1}(\mathbb{R})}<\infty .
$$

In view of identity (0.4), this clearly measures some features of the smoothness of the function $u(\cdot)$. In fact, it combines regularity and geometrical aspects. For instance, the expression $\tilde{u}(\omega, \cdot)$ associated with an application $u(\cdot)$ whose leaves are transversal to $\omega$ :

$$
\begin{aligned}
& u=\bar{u} \circ \varphi ; \quad(\bar{u}, \varphi) \in L^{\infty}(\mathbb{R}) \times C^{1}\left(\mathbb{R}^{N} ; \mathbb{R}\right) ; \\
& 0 \neq \nabla \varphi(x) \not \mid \omega, \quad \forall x \in \mathbb{R}^{N},
\end{aligned}
$$

is subjected to (5.1). 


\subsection{A two-microlocal interpretation}

We now proceed to interpret inequality (4.4) in the multidimensional setting from the point of view of the initial variables:

PROPOSITION 5.2.1.-

We consider the transport equation $\left(\mathcal{T}_{0}^{N}\right)$ associated with some initial data $g_{0}(\cdot)$ chosen in the space $\mathcal{M}_{b}^{m, n}\left(\mathbb{R}_{x}^{N} \times \mathbb{R}_{v}\right)$ with compact support included in the strip $\mathbb{R} \times\left[-\varrho_{0}^{\infty}, \varrho_{0}^{\infty}\right]$. Then, for any decomposition of $\mathbb{R}_{x}^{N}$ into a family of parallel hyperplanes with normal vector $\omega$, we get an inequality:

$$
\begin{aligned}
& \left\|\int_{\mathbb{R}}\left(\omega \cdot a^{\prime}(v)\right)^{2 n-1} g_{0}(\cdot-s a(v), v) d v\right\|_{H^{\tau, \bar{\tau}}\left(\mathbb{R}_{y^{\prime}}^{1} \times \mathbb{R}_{y^{\prime \prime}}^{N-1}\right)} \\
& \quad \leqslant C\left(m, n, a, b_{n}\right)\left(1+s^{n}\right)\left\|g_{0}\right\|_{\mathcal{M}_{b}^{m, n}\left(\mathbb{R}_{x}^{N} \times \mathbb{R}_{v}\right)} / s^{n}, \quad \forall s \in \mathbb{R}_{*}^{+},
\end{aligned}
$$

which is true for all indexes $\tau$ and $\bar{\tau}$ limited by:

$$
\tau<m+n-\frac{1}{2}, \quad \bar{\tau}<-n+\frac{1-N}{2} .
$$

Proof of Proposition 5.2.1. - For definiteness, we will work with vector:

$$
\omega=\omega_{1}=(1,0, \ldots, 0)
$$

and the corresponding decomposition

$$
\mathbb{R}_{x}^{N} \ni x=\left(x_{1},\left(x_{2}, \ldots, x_{N}\right)\right)=\left(z, x^{\prime \prime}\right) \in \mathbb{R}_{z}^{1} \times \mathbb{R}_{x^{\prime \prime}}^{N-1}
$$

with dual variables

$$
T^{*}\left(\mathbb{R}_{x}^{N}\right) \ni \xi=\left(\xi^{\prime}, \xi^{\prime \prime}\right) \in \mathbb{R}_{\xi^{\prime}}^{1} \times \mathbb{R}_{\xi^{\prime \prime}}^{N-1}
$$

We shall designate $\ddot{a}(v):=\left(a_{2}(v), \ldots, a_{N}(v)\right)$ the $N-1$ last components of the speed. We have the relation:

$$
\begin{array}{r}
\mathcal{F}_{q}\left(\int_{\mathbb{R}} a_{1}(v)^{2 n-1} g_{0}(\cdot-s a(v), v) d v\right)(\xi) \\
=\mathcal{F}_{1}\left(\int_{\mathbb{R}} k^{s, \xi^{\prime \prime}}\left(\cdot-s a_{1}(v), v\right) d v\right)\left(\xi^{\prime}\right) .
\end{array}
$$


Now, the proof is imbued from the one given in P. Gérard [11]. The expression $k^{s, \xi^{\prime \prime}}(\cdot)$ introduced in (5.4) has parameters $s$ and $\xi^{\prime \prime}$. After a short calculation, we obtain:

$$
k^{s, \xi^{\prime \prime}}(z, v)=b_{n}(v) a_{1}(v)^{2 n-1} \bar{k}_{n}^{s, \xi^{\prime \prime}}(z, v),
$$

where:

$$
\begin{gathered}
b_{n} \in C_{c}^{n}(\mathbb{R}), \quad b_{n}(v)=1, \quad \forall v \in\left[\varrho_{0}^{\infty}, \varrho_{0}^{\infty}\right] . \\
\bar{k}_{n}^{s, \xi^{\prime \prime}}(z, v):=\int_{\mathbb{R}^{N-1}} \mathrm{e}^{-i x^{\prime \prime} \cdot \xi^{\prime \prime}} \mathrm{e}^{-i s \ddot{a}(v) \cdot \xi^{\prime \prime}} g_{0}\left(z, x^{\prime \prime}, v\right) d x^{\prime \prime} .
\end{gathered}
$$

We select $(i, j)$ in $[0, m] \times[0, n]$ and exploit the identity

$$
\partial_{z}^{i} \partial_{v}^{j} \bar{k}_{n}^{s, \xi^{\prime \prime}}(z, v)=\int_{\mathbb{R}^{N-1}} \mathrm{e}^{-i x^{\prime \prime} \cdot \xi^{\prime \prime}} \partial_{v}^{j}\left\{\mathrm{e}^{-i s \ddot{a}(v) \cdot \xi^{\prime \prime}} \partial_{z}^{i} g_{0}\left(z, x^{\prime \prime}, v\right)\right\} d x^{\prime \prime},
$$

to get:

$$
\left|\bar{k}_{n}^{s, \xi^{\prime \prime}}\right|_{\mathcal{M}_{b}^{i, j}\left(\mathbb{R}_{z} \times \mathbb{R}_{v}\right)} \leqslant C(A)\left(1+s^{j}\right)\left\langle\xi^{\prime \prime}\right\rangle_{N-1}^{j}\left\|g_{0}\right\|_{\mathcal{M}_{b}^{m, n}\left(\mathbb{R}_{x}^{N} \times \mathbb{R}_{v}\right)} .
$$

It follows that the Cauchy data $k^{s, \xi^{\prime \prime}}(\cdot)$ is well prepared up to the order $(m, n)$ for the flux $A_{1}(\cdot)$. Therefore we can apply Proposition 4.1 which implies:

$$
\begin{aligned}
& \left\|\int_{\mathbb{R}} k^{s, \xi^{\prime \prime}}\left(\cdot-s a_{1}(v), v\right) d v\right\|_{\mathcal{M}_{b}^{m+n}(\mathbb{R})} \\
& \quad \leqslant C\left(m, n, a, b_{n}\right) \frac{1+s^{n}}{s^{n}}\left\langle\xi^{\prime \prime}\right\rangle_{N-1}^{n}\left\|g_{0}\right\|_{\mathcal{M}_{b}^{m, n}\left(\mathbb{R}_{x}^{N} \times \mathbb{R}_{v}\right)} .
\end{aligned}
$$

A classical Sobolev inclusion says that:

$$
\|u\|_{H^{\tau}(\mathbb{R})} \leqslant C\|u\|_{\mathcal{M}_{b}^{m+n}(\mathbb{R})}, \quad \forall \tau<m+n-\frac{1}{2} .
$$

Upon combining informations (5.4), (5.5) and (5.6), we conclude that for every parameter $\tau$ selected according to (5.6) we have:

$$
\begin{aligned}
& \left\|\left\langle\xi^{\prime}\right\rangle_{1}^{\tau} \mathcal{F}_{q}\left(\int_{\mathbb{R}} a_{1}(v)^{2 n-1} g_{0}(\cdot-s a(v), v) d v\right)\left(\xi^{\prime}, \xi^{\prime \prime}\right)\right\|_{L^{2}\left(\mathbb{R}_{\xi^{\prime}}\right)} \\
& \leqslant C\left(m, n, a, b_{n}\right) \frac{1+s^{n}}{s^{n}}\left\langle\xi^{\prime \prime}\right\rangle_{N-1}^{n}\left\|g_{0}\right\|_{\mathcal{M}_{b}^{m, n}\left(\mathbb{R}_{x}^{N} \times \mathbb{R}_{v}\right)} .
\end{aligned}
$$


Finally, we multiply the left hand side by a power $\left\langle\xi^{\prime \prime}\right\rangle^{\bar{\tau}}$ where the real number $\bar{\tau}$ is adjusted in such a way to win the $L^{2}\left(\mathbb{R}_{\xi^{\prime \prime}}^{N-1}\right)$ integrability with respect to $d \xi^{\prime \prime}$. This criterion determines the bound $\bar{\tau}$ imposed in (5.2).

By applying to function $\Xi_{t}^{N}\left(\varrho_{0}\right)(\cdot)$ Proposition 5.3.1 with the particular choice $(m, n)=(0,1)$, we obtain inequality (2.22). The proof of Proposition 2.2.1 is complete.

\subsection{Miscellaneous results}

As an aside, we first consider the large time behavior of periodic solutions. This subject has been studied in Dafermos [8], Lyberopoulos [20] and Engquist-W. E [10]. The question is to know if the solution $\varrho(t, \cdot)$ tends to some constant state.

It turns out that the demonstrations of Theorems 1.1 and 1.2 yield a number $\Theta\left(t, \varrho_{0}\right)$ which does not depend on $t$. This particularity gives a powerful tool regarding the preceding preoccupation. It suffices to exploit Theorems 1.1, 1.2, 1.3 and the inversion formula (given p. 72 in Helgason [13]).

We can assert:

COROLlARY 5.3.1 (Large time periodic behavior). - Let $\varrho(\cdot)$ be an admissible periodic solution of $\left(\mathcal{L}_{0}^{N}\right)$ with $A(\cdot)$ satisfying the hypothesis of Theorem 1.1 (if $N=1$ ) or the hypothesis of Theorem 1.2 (if $N \geqslant 2$ ). Then:

$$
\begin{aligned}
& \exists C(A, b) \in \mathbb{R}^{+} ; \forall\left(\omega, B, \varrho_{0}\right) \in \mathbb{S}^{N-1} \times C_{A}^{\omega}(\mathbb{R}) \times L^{\infty}\left(\mathbb{T}^{N}\right), \\
& \forall t \in \mathbb{R}_{*}^{+},\|B \circ \varrho(t, \cdot)\|_{\mathcal{M}_{b, \omega}^{1}(\mathbb{T})} \leqslant C(A, b)\left(1+\left\|\varrho_{0}\right\|_{L^{1}\left(\mathbb{T}^{N}\right)}\right) / t .
\end{aligned}
$$

As noted by Zumbrun (see (2.5) in Theorem 2.1 of [30], the most natural way to tackle the non convex case is in deriving estimates in terms of the total variation of the wave speed. This (already mentioned) fact can be recovered in our approach since function $a(\cdot)$ belongs systematically to the allowed space $C_{A}^{1}(\mathbb{R})$. Combining Theorems 1.1 and 1.3 , we can improve the result of Zumbrun [30]:

COROLLARY 5.3.2 (smoothing of the wave speed).-Assume $(\mathcal{I})^{1}$. Then:

$$
\begin{aligned}
& \forall t \in \mathbb{R}_{*}^{+}, \forall \varrho_{0} \in L_{c}^{\infty}(\mathbb{R}), \\
& \quad\|a \circ \varrho(t, \cdot)\|_{B V(\mathbb{R})} \leqslant C(A)\left(\left|\operatorname{supp} \varrho_{0}+t V\right|+\left\|\varrho_{0}\right\|_{L^{1}(\mathbb{R})}\right) / t .
\end{aligned}
$$


Other non linear expressions have an interest. Let us introduce:

$$
\bar{b} \in C^{0}\left(\mathbb{R}_{*}\right), \quad \bar{b}(\varrho):=a(\varrho) / a^{\prime}(\varrho), \quad \forall \varrho \in \mathbb{R}_{*} .
$$

Under the more restrictive assumption (2.19), function $\bar{b}(\cdot)$ can be extended to all the $\varrho$-axis in such a way that $\bar{b}(\cdot) \in \operatorname{Lip}_{c}(\mathbb{R})$. Therefore, we can apply Theorem 1.3. We deduce:

COROLlary 5.3.3 (time smoothing effect). - Assume (2.19). Then, $\varrho(\cdot)$ belongs to the space $\operatorname{Lip}_{\text {loc }}\left(\mathbb{R}_{*}^{+} ; L^{1}(\mathbb{R})\right)$.

Proof of Corollary 5.3.3. - The expression $\bar{B}(\cdot)$ corresponding to $\bar{b}(\cdot)$ is exactly the flux function $A(\cdot)$ since:

$$
\bar{B}(\varrho):=\int_{-\infty}^{\varrho} \bar{b}(v) a^{\prime}(v) d v=\int_{-\infty}^{\varrho} a(v) d v=A(\varrho) .
$$

Using Theorem 1.3, keeping in mind equation $\left(\mathcal{L}_{0}^{1}\right)$, we obtain:

$$
\begin{aligned}
\left\|u\left(t_{2}, \cdot\right)-u\left(t_{1}, \cdot\right)\right\|_{L^{1}(\mathbb{R})} \leqslant & \int_{t_{1}}^{t_{2}}\left(\int_{\mathbb{R}}\left|\partial_{x}[A(\varrho)](s, x)\right| d x\right) d s \\
\leqslant & C\left(t_{1}, t_{2}, A, \varrho_{0}\right)\left|t_{2}-t_{1}\right|<\infty, \\
& \forall\left(t_{1}, t_{2}\right) \in\left(\mathbb{R}_{*}^{+}\right)^{2} . \quad \square
\end{aligned}
$$

Remark 5.1. - The topics of time regularizing effects has already been treated. Using an elegant scaling argument Benilan and Crandall [1] show that in the homogeneous case $\left(A(\lambda \varrho)=\lambda^{m+1} A(\varrho)\right)$ solutions of $\left(\mathcal{L}_{0}^{1}\right)$ do satisfy:

$$
\lim _{h \rightarrow 0} \frac{1}{h}\|\varrho(t+h, \cdot)-\varrho(t, \cdot)\|_{L^{1}(\mathbb{R})} \leqslant \frac{2}{m t}\left\|\varrho_{0}\right\|_{L^{1}(\mathbb{R})}, \quad \forall t \in \mathbb{R}_{*}^{+} .
$$

In the absence of homogeneity, the strongest result of this type is due to F. Otto [23]. This author considers the situation (2.19) and exhibits some Hölder exponent:

$$
\varrho \in C^{\alpha}\left(\mathbb{R}_{*} ; L^{1}\left(\mathbb{R}^{N}\right)\right), \quad \alpha:=1 / m \leqslant 1 / 3<1 .
$$

His method is based on the $L^{1}$ contraction principle. Therefore, the smoothing effect is controlled by the $L^{1}$ norm of $\varrho_{0}(\cdot)$ and nothing else. 
This restriction is at the origin of the bound given for $\alpha$. In Dafermos [8], this was the reason of the occurrence of the conjugate function $D(\cdot)$. Indeed, Dafermos comes back to zero with the help of its formula (2.9)[8] that produces the $L^{1}$ norm of $\varrho_{0}(\cdot)$ but also, as a consequence, the expression $D(\cdot)$. We adopt a slightly different point of view: we have more freedom for $\alpha$ and for the allowed non linear compositions on condition that we incorporate in the right hand of the estimations the measure of the support of $\varrho_{0}(\cdot)$.

We investigate now the level of Sobolev regularity recovered at time $t>0$ by the average solution of $\left(\mathcal{T}_{0}^{N}\right)$ :

$$
\varrho(t, \cdot):=\int_{\mathbb{R}} g(t, \cdot, v) d v \in W^{\tau, 1}\left(\mathbb{R}^{N}\right), \quad \forall \tau \in[0, \bar{\tau}[,
$$

when working under $(\mathcal{N S})^{N}$ and data subjected to:

$$
g(t, x, v)=\chi_{\varrho(t, x)}(v), \quad\|g(s, \cdot)\|_{\mathcal{M}_{b}^{0,1}\left(\mathbb{R}_{x}^{N} \times \mathbb{R}_{v}\right)}<\infty, \quad \forall s \in[0, t],
$$

which is guaranteed once we have $\Theta\left(t, \varrho_{0}\right)<\infty$.

COROLlary 5.3.4 (Sobolev regularity). - Assume the hypothesis of Theorems 1.1 or 1.2 and compute the index $\alpha$ involved in $(\mathcal{N S})^{N}$ according to Remark 2.2.2. Then:

$$
\begin{aligned}
& \varrho(t, \cdot) \in W^{\tau, 2}\left(\mathbb{R}_{x}^{N}\right), \quad \forall \tau<\alpha / 2, \forall t \in \mathbb{R}_{*}^{+} . \\
& \varrho(t, \cdot) \in W^{\tau, 1}\left(\mathbb{R}_{x}^{N}\right), \quad \forall \tau<2 \alpha / 3, \forall t \in \mathbb{R}_{*}^{+} .
\end{aligned}
$$

Proof of Corollary 5.3.4. - The demonstration relies on the work of [18] and on a remark due to F. Bouchut (see [2] and [3]). We first multiply $g(\cdot)$ by a cutoff function $\chi(\cdot) \in C_{c}^{\infty}\left(\mathbb{R}_{s}\right)$ with $\chi \equiv 1$ in a neighborhood of $t$. It yields $(\chi g)(\cdot)$ in $L_{c}^{\infty}\left(\mathbb{R}_{t} \times \mathbb{R}_{x}^{N} \times \mathbb{R}_{v}\right)$.

Let $\psi(\cdot)$ satisfy:

$$
\psi(\cdot) \in C_{c}^{\infty}\left(\mathbb{R}_{v}\right), \quad 0 \leqslant \psi(v) \leqslant 1, \forall v \in \mathbb{R}, \int_{\mathbb{R}} \psi(v) d v=1
$$

and set for $\varepsilon>0: \psi_{\varepsilon}(v):=\psi(v / \varepsilon) / \varepsilon$. We can decompose $g(\cdot)$ into:

$$
g(s, x, v)=\left(g *_{v} \psi_{\varepsilon}\right)(s, x, v)+\left(g-g *_{v} \psi_{\varepsilon}\right)(s, x, v),
$$


where the convolution $*_{v}$ concerns only the variable $v$. The idea is to evaluate the first part $\left(g *_{v} \psi_{\varepsilon}\right)(\cdot)$ thanks to the averaging lemma (in a version given by Bouchut [3]) and the second part $\left(g-g *_{v} \psi_{\varepsilon}\right)(\cdot)$ by using the a priori estimate (5.9).

By applying the Proposition 5.1 line (5.24) of [3] to the equation:

$$
\partial_{s}(\chi g)(s, x, v)+a(v) \cdot \nabla_{x}(\chi g)(s, x, v)=\chi^{\prime}(s) g(s, x, v),
$$

we obtain:

$$
\begin{aligned}
& \left\|\chi g *_{v} \psi_{\varepsilon}\right\|_{L^{2}\left(\mathbb{R}_{t} \times \mathbb{R}_{v} ; \dot{W}^{\alpha / 2,2}\left(\mathbb{R}_{x}^{N}\right)\right)} \\
& \quad \leqslant C\left(\|\chi g\|_{L^{2}\left(\mathbb{R}_{t} \times \mathbb{R}_{x}^{N} \times \mathbb{R}_{v}\right)}+\left\|\chi^{\prime} g\right\|_{L^{2}\left(\mathbb{R}_{t} \times \mathbb{R}_{x}^{N} \times \mathbb{R}_{v}\right)}\right) / \sqrt{\varepsilon} .
\end{aligned}
$$

Since $\chi^{\prime}(\cdot)$ is null on some open interval containing $\{t\}$, the evolution equation reduces near the time $\{t\}$ to the free transport $\left(\mathcal{T}_{0}^{N}\right)$ which (by interpolation) preserves the $L^{2}\left(\mathbb{R}_{v} ; \dot{W}^{\alpha / 2,2}\left(\mathbb{R}_{x}^{N}\right)\right)$-norm. This argument allows to extract from (5.12) an information for the trace at time $t$ :

$$
\left\|g *_{v} \psi_{\varepsilon}(t, \cdot)\right\|_{L^{2}\left(\mathbb{R}_{v} ; \dot{W}^{\alpha / 2,2}\left(\mathbb{R}_{x}^{N}\right)\right)} \leqslant C / \sqrt{\varepsilon} .
$$

Combining (5.6) and (5.9), we find:

$$
\left\|\left(g-g *_{v} \psi_{\varepsilon}\right)(t, \cdot)\right\|_{L^{2}\left(\mathbb{R}_{x}^{N} \times \mathbb{R}_{v}\right)} \leqslant \varepsilon^{\beta}, \quad \forall \beta<1 / 2 .
$$

Let $\tau_{h}$ be the translation operator in space variable:

$$
\left(\tau_{h} g\right)(t, x, v):=g(t, x+h, v), \quad h \in \mathbb{R}_{x}^{N} .
$$

We apply $\tau_{h}-I$ to the line (5.10) and use the informations (5.13) and (5.14) to get:

$$
\left\|\left(\tau_{h} g-g\right)(t, \cdot)\right\|_{L^{2}\left(\mathbb{R}_{x}^{N} \times \mathbb{R}_{v}\right)} \leqslant C\left(|h|^{\alpha / 2} / \sqrt{\varepsilon}+2 \varepsilon^{\beta}\right), \quad \forall \beta<1 / 2 .
$$

We adjust parameter $\varepsilon$ in an optimal way to find:

$$
g(t, \cdot) \in L^{2}\left(\mathbb{R}_{v} ; \dot{W}^{s_{0}, 2}\left(\mathbb{R}_{x}^{N}\right)\right), \quad \forall s_{0}<\alpha / 4 .
$$

We apply again the averaging lemma with $\left\langle D_{x}\right\rangle^{s_{0}} g(\cdot)$ in place of $g(\cdot)$ to find:

$$
\left\|\left\langle D_{x}\right\rangle^{s_{0}} g *_{v} \psi_{\varepsilon}(t, \cdot)\right\|_{L^{2}\left(\mathbb{R}_{v} ; \dot{W}^{\alpha / 2,2}\left(\mathbb{R}_{x}^{N}\right)\right)} \leqslant C / \sqrt{\varepsilon} .
$$


Reiterating the preceding argument, we deduce that $g(t, \cdot)$ belongs to the space:

$$
g(t, \cdot) \in L^{2}\left(\mathbb{R}_{v} ; \dot{W}^{s_{1}, 2}\left(\mathbb{R}_{x}^{N}\right)\right), \quad \forall s_{1}<s_{0} / 2+\alpha / 4
$$

Since $\alpha / 2$ is the unique fixed point of the relation $s \mapsto s / 2+\alpha / 4$, we have:

$$
g(t, \cdot) \in L^{2}\left(\mathbb{R}_{v} ; \dot{W}^{s, 2}\left(\mathbb{R}_{x}^{N}\right)\right), \quad \forall s<\alpha / 2,
$$

which yields the first part of Corollary 5.3.4.

Following the same principle, we also have (for all $\bar{\alpha}<\alpha$ ):

$$
\begin{aligned}
& \left\|\left(\tau_{h} g-g\right)(s, \cdot)\right\|_{L^{1}\left(\mathbb{R}_{x}^{N} \times \mathbb{R}_{v}\right)} \\
& \quad \leqslant\left\|D_{x}^{\bar{\alpha}} g * \psi_{\varepsilon}\right\|_{L^{1}\left(\mathbb{R}_{x}^{N} \times \mathbb{R}_{v}\right)}|h|^{\bar{\alpha}}+2\left\|g-g * \psi_{\varepsilon}\right\|_{L^{1}\left(\mathbb{R}_{x}^{N} \times \mathbb{R}_{v}\right)} \\
& \quad \leqslant\left\|D_{x}^{\bar{\alpha} / 2}\left(D_{x}^{\bar{\alpha} / 2} g\right) * \psi_{\varepsilon}\right\|_{L^{2}\left(\mathbb{R}_{x}^{N} \times \mathbb{R}_{v}\right)}|h|^{\bar{\alpha}}+2 \varepsilon\left\|\partial_{v} g(s, \cdot)\right\|_{\mathcal{M}_{b}\left(\mathbb{R}_{x}^{N} \times \mathbb{R}_{v}\right)} \\
& \quad \leqslant C|h|^{\bar{\alpha}} / \sqrt{\varepsilon}+2 \varepsilon \Theta\left(s, \varrho_{0}\right) .
\end{aligned}
$$

We just have to set $\varepsilon=|h|^{2 \bar{\alpha} / 3}$ to conclude.

\section{REFERENCES}

[1] Bénilan P., Crandall M.G., Regularizing effects of homogeneous evolution equations, in: Contributions to Analysis and Geometry, John Hopkins Univ. Press, Baltimore MD, 1981, pp. 23-39.

[2] Bouchut F., Desvillettes L., Averaging lemmas without time Fourier transform and applications to discretized kinetic equations, Proc. Royal Soc. Edinburgh A 129 (1999) 19-36.

[3] Bouchut F., Introduction to the mathematical theory of kinetic equations, in: Coll. Series in Appl. Math. Session "L'état de la recherche" de la S. M. F., Equations cinétiques, Orléans, Elsevier, Amsterdam, 1998.

[4] Brenier Y., Averaged multivalued solutions fot scalar conservation laws, SIAM J. Numer. Anal. 6 (1984) 1013-1037.

[5] Cheverry C., Effet régularisant pour une loi de conservation scalaire multidimensionnelle, Séminaire: Equations aux derivees partielles, 1998-99, Exp. No. XXIV, 15 pp., Ecole Polytechnique, Palaiseau.

[6] Conway E.D., The formation and decay of shocks for a conservation law in several dimensions, Arch. Rational Mech. Anal. 64 (1977) 47-57.

[7] Dafermos C., Asymptotic behavior of solutions of hyperbolic balance laws, in: Bardos C., Bessis D. (Eds.), Bifurcation Phenomena in Mathematical Physics, Reidel, Dordrecht, 1979, pp. 521-533.

[8] Dafermos C., Regularity and large time behaviour of solutions of a conservation law without convexity, Proc. Roy. Soc. Edinburgh Sect. A 99 (1985) 201-239. 
[9] DiPerna R.J., Lions P.L., Meyer Y., $L^{p}$ regularity of velocity averages, Ann. Inst. Henri Poincaré 8 (1991) 271-287.

[10] Engquist B., E W., Large time behavior and homogenization of solutions of twodimensional conservation laws, Comm. Pure Apl. Math. 46 (1993) 1-26.

[11] Gérard P., Moyennisation et régularité deux-microlocale, Ann. Sci. Ecole Norm. Sup. 23 (1990) 89-121.

[12] Golse F., Lions P.L., Perthame B., Sentis R., Regularity of the moments of the solution of a transport equation, J. Funct. Anal. 76 (1988) 110-125.

[13] Helgason S., The Radon Transform, Progress in Mathematics, Vol. 5, Birkhäuser.

[14] Kruzkov S.N., First-order quasilinear equations in several independent variables, Math. USSR-Sb. 64 (1977) 47-57.

[15] Lax P.D., Hyperbolic systems of conservation laws and the mathematical theory of shock waves, in: Regional Conference Series in Applied Mathematics, SIAM, 1973.

[16] Lax P.D., The formation and decay of shock waves, Amer. Math. Month. 3 (1972) 227-241.

[17] Liu T.P., Pierre M., Source solutions and asymptotic behavior in conservation laws, SIAM J. Math. Anal. 19 (1988) 763-773.

[18] Lions P.L., Perthame B., Tadmor E., A kinetic formulation of multidimensional scalar conservation laws and related equations, Bull. Amer. Math. Soc. 7 (1994) 169-189.

[19] Lucier B.J., Regularity through approximation for scalar conservation laws, Comm. Pure Appl. Math. 10 (1957) 537-566.

[20] Lyberopoulos N., A Poincaré-Bendixon theorem for scalar balance laws, Proc. Royal Soc. Edinburgh A 124 (1994) 589-607.

[21] Murat F., Compacité par compensation, Ann. Scuola Norm. Sup. Pisa Cl. Sci. 5 (1978) 489-507.

[22] Oleinik O., Discontinuous solutions of nonlinear differential equations, Usp. Mat. Nauk. 26 (1963) 95-172.

[23] F. Otto, A regularizing effect of nonlinear transport equations, Quart. Appl. Math. 56 (1998) 355-375.

[24] Perthame B., Tadmor E., A kinetic equation with kinetic entropy functions for scalar conservation laws, Comm. Math. Phys. 136 (1991) 501-517.

[25] Schaeffer D.G., A regularity theorem for conservation laws, Adv. in Math. 11 (1973) 368-386.

[26] Serre D., Systèmes de lois de conservation I, Diderot éditeur, arts et sciences.

[27] Tartar L., Une nouvelle méthode de résolution d'équations aux dérivées partielles non linéaires, in: Lecture Notes in Math., Vol. 665, Springer, Berlin, 1977, pp. 228 241.

[28] Vasseur A., Contributions à l'approche cinétique des systèmes de lois de conservation hyperboliques, Thèse de doctorat de l'université Paris 6, 1999.

[29] Volpert A.I., The space BV and quasilinear equations, Mat. Sb. 73 (1967). English translation: Math. USSR Sb. 2 (1967) 225-267.

[30] Zumbrun K., Decay rates for nonconvex systems of conservation laws, Comm. Pure Appl. Math. 46 (1993) 353-386. 